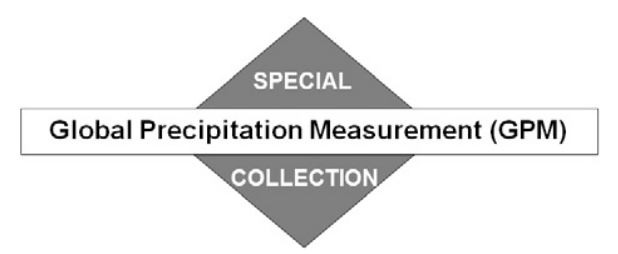

\title{
Physical Evaluation of GPM DPR Single- and Dual-Wavelength Algorithms
}

\author{
LIANG LIAO \\ Goddard Earth Science Technology and Research, Morgan State University, Greenbelt, Maryland \\ ROBERT MENEGHINI \\ NASA Goddard Space Flight Center, Greenbelt, Maryland
}

(Manuscript received 16 November 2018, in final form 21 March 2019)

\begin{abstract}
A physical evaluation of the rain profiling retrieval algorithms for the Dual-Frequency Precipitation Radar (DPR) on board the Global Precipitation Measurement (GPM) Core Observatory satellite is carried out by applying them to the hydrometeor profiles generated from measured raindrop size distributions (DSD). The DSD-simulated radar profiles are used as input to the algorithms, and their estimates of hydrometeors' parameters are compared with the same quantities derived directly from the DSD data (or truth). The retrieval accuracy is assessed by the degree to which the estimates agree with the truth. To check the validity and robustness of the retrievals, the profiles are constructed for cases ranging from fully correlated (or uniform) to totally uncorrelated DSDs along the columns. Investigation into the sensitivity of the retrieval results to the model assumptions is made to characterize retrieval uncertainties and identify error sources. Comparisons between the single- and dual-wavelength algorithm performance are carried out with either a single- or dualwavelength constraint of the path integral or differential path integral attenuation. The results suggest that the DPR dual-wavelength algorithm generally provides accurate range-profiled estimates of rainfall rate and mass-weighted diameter with the dual-wavelength estimates superior in accuracy to those from the singlewavelength retrievals.
\end{abstract}

\section{Introduction}

The Dual-Frequency Precipitation Radar (DPR) operating at $\mathrm{Ku}$ and $\mathrm{Ka}$ band, on board the Global Precipitation Measurement (GPM) Core Observatory satellite, provides estimates of rainfall rate and parameters of the raindrop size distribution (DSD) (Hou et al. 2014; Skofronick-Jackson et al. 2017, 2018). To obtain parameters of the DSD, it is often modeled either as a gamma (Ulbrich 1983; Gorgucci et al. 2000, 2002; Bringi et al. 2002) or lognormal distribution (Feingold and Levin 1986), and several dual-wavelength radar retrieval techniques have been developed (Meneghini et al. 1992; Mardiana et al. 2004; Liao and Meneghini 2005; Rose and Chandrasekar 2005; Seto et al. 2013; Seto and Iguchi 2015; Liao et al. 2016; Liao and Meneghini 2019). Some of them are based on an analytic technique that uses the dual-frequency ratio (DFR), that is, the difference of radar reflectivities between two wavelengths, to estimate a

Corresponding author: Dr. Liang Liao, liang.liao-1@ nasa.gov characteristic size parameter of the distribution, with the second measurement giving an estimate related to the number concentration (Meneghini et al. 1992, 1997; Mardiana et al. 2004; Liao and Meneghini 2005; Rose and Chandrasekar 2005; Seto et al. 2013; Liao et al. 2016). Other approaches rely on an optimization method in which one or more adjustment factors are used to modify some nominal relationships between the parameters of the radar and the precipitation to account for variations in DSD in space and time (Grecu et al. 2011; Seto and Iguchi 2015; Liao and Meneghini 2019). Adjustment factors at each gate/profile are determined by optimizing predefined cost functions that constrain radar measurements.

Early versions of the DPR algorithms, such as version-3 algorithms, rested on the DFR-based technique, which is, in principle, capable of fully characterizing spatial and temporal DSD variations. However, because of the fact that dual solutions exist in estimating mass-weighted diameter $\left(D_{m}\right)$ from DFR for small values of $D_{m}$, ambiguities in the estimation of DSD often occur, particularly for light-to-moderate rain rates. As there is no 
additional information aiding in selection of the solution, the DFR-based technique becomes problematic for rain retrievals; moreover, retrieval errors caused by an incorrect choice of solution worsen as the retrieval proceeds into the storm because of error propagation.

To circumvent the limitations encountered by the DFR-based technique, the current DPR-operational algorithm adopts an optimization approach that is based on a relationship between rain rate $R$ and $D_{m}$, that is, $R-D_{m}$ relation. The constraint of an $R-D_{m}$ relation implies that the gamma DSD has only one free parameter, such as $D_{m}$, if its shape factor is either fixed or expressed as a function of $D_{m}$. Thus, one is able to relate the radar reflectivity to $D_{m}$. An adjustment factor is used to modify $R-D_{m}$ relation for each vertical hydrometeor profile. A search for the adjustment factor is conducted by minimizing differences between simulated and measured radar reflectivities as well as the path integral attenuation (PIA) in the case of a single wavelength and differential PIA ( $\delta$ PIA) for dual wavelengths. Once the adjustment factor is found, the DSD and rain rate can be uniquely derived along each profile. An obvious advantage of this optimal approach is to avoid retrieval uncertainties arising from the double solutions such as those that occur from the use of the DFR. On the other hand, this approach loses the capability of fully accounting for the DSD variations along the profile because a single adjustment factor is not able to account for variations in the DSD along the profile. In fact, the performance depends on a number of factors that include the model assumptions and the degree of uniformity of the DSD along the profiles. An evaluation of the algorithm performance is important in assessing the strengths and weaknesses of the algorithm and in gaining insight into ways to improve it.

Simultaneous comparisons of collocated PR and DPR estimates with the similar quantities derived from the ground measurements, such as radars and rain gauge networks, provide direct checks of the PR/DPR algorithms (Liao et al. 2001; Wolff et al. 2005; Liao and Meneghini 2009a,b; Schwaller and Morris 2011). This is in fact a very important task for the validation of the DPR products, and many efforts have been put into such studies. However, because of different beamwidths, scanning geometries and possible temporal offsets, the space- and ground-radar scattering volumes cannot be perfectly matched. In addition, the issue of nonuniform beam filling (NUBF) across $5 \mathrm{~km} \times 5 \mathrm{~km}$ DPR footprint further complicates the algorithm evaluation. While direct comparisons between space-based and ground-based sensors remain essential, they do not fully meet the need for algorithm testing and improvement. To validate the algorithm principles and their assumptions, a physical and realistic framework/test bed is employed to enable an accurate assessment of the performance of the operational algorithms.

To achieve this, measured time series DSD data are used in this study to construct vertical rain profiles. With the known profile of particle size distribution and particle phase state, the true and measured reflectivity factors as well as PIA can be computed from forward scattering models. The Ku- and Ka-band measured reflectivity profiles can then be used as input to the retrieval algorithms. The degree to which the radar estimates agree with the true values, which are derived directly from the assumed DSD profiles, constitutes a measure of the retrieval accuracy. The basic approach can also be used to evaluate the impact of different model assumptions and various constraints adopted in the retrieval algorithms.

In this paper we start with describing the principles of the DPR version-5 algorithms in section 2, and then evaluate its accuracy in section 3 with respect to several key factors that affect the performance. Summary and remarks are provided in section 4.

It is important to note that what is called the DPR algorithm in this paper should be clearly distinguished from the operational version of the algorithm. The operational algorithm, for example, must deal with mixed phase hydrometeors and snow as well as corrections for cloud water and atmospheric gases. Our purpose rather is to understand the performance of the basic optimization approach used in the algorithm and how its accuracy is affected by characteristics of the DSD along the path and in how the various constraints influence the choice of solution.

\section{DPR algorithm}

The version-5 DPR algorithms, both single and dual wavelength, adopt an optimization approach that is based on a relationship between rain rate and massweighted diameter, that is, the $R-D_{m}$ relation. This can be considered an evolution from the single-wavelength algorithm devised for application to data from the Precipitation Radar (PR) on the Tropical Rainfall Measuring Mission (TRMM) satellite (Iguchi et al. 2000, 2009). The PR algorithm relies on a modification to the $k-Z$ relation, where $k$ is specific attenuation and $Z$ is radar reflectivity factor, by a multiplicative $\varepsilon$ factor that is used to adjust the initial $k-Z$ relationship toward one that satisfies an independent path attenuation constraint (Meneghini et al. 1983; Iguchi and Meneghini 1994). A similar kind of $\varepsilon$ adjustment is used in the DPR algorithm but applied to the $R-D_{m}$ relationship. A search for the optimum adjustment factor is conducted by minimizing differences between the simulated and measured radar reflectivities as well as between the 
measured and DSD-derived values of PIA or $\delta$ PIA. Once the adjustment factor is found, the DSD and rain rate can be derived along each profile. Its performance depends on a number of factors that include the model assumptions, the type of constraints and the nonuniformity of hydrometeor profiles.

Explicitly, the $R-D_{m}$ can be written in the form

$$
R=\varepsilon^{\tau} a D_{m}^{b},
$$

where $a, b$, and $\tau$ are constants. For the current operational DPR algorithms, these values, obtained from the DSD measurements (Kozu et al. 2009), are, respectively, $0.401,6.131$, and 4.649 for stratiform rain and 1.370 , 5.420 , and 4.258 for convective rain. Rain rate $R$ is in units of $\mathrm{mm} \mathrm{h}^{-1}$ while $D_{m}$ is in $\mathrm{mm}$. Parameter $\varepsilon$ is defined as the same modification factor to the $k-Z$ relation used for the single-wavelength algorithm (Meneghini et al. 1983; Iguchi and Meneghini 1994; Kozu et al. 2009), such as TRMM PR. It is, however, adjusted for each vertical rain profile to obtain solutions to the radar retrieval equations that, as discussed below, minimize the predefined cost function.

If the DSD, $N(D)\left(\mathrm{m}^{-3} \mathrm{~mm}^{-1}\right)$, is expressed as a normalized gamma form (Testud et al. 2001; Bringi and Chandrasekar 2001; Bringi et al. 2002; Chandrasekar et al. 2005; Seto et al. 2013; Seto and Iguchi 2015; Williams et al. 2014), which, in terms of volume-equivalent diameter $D(\mathrm{~mm})$, is given by

$$
\begin{aligned}
N(D) & =N_{w} f(\mu)\left(\frac{D}{D_{m}}\right)^{\mu} \exp (-\Lambda D), \\
f(\mu) & =\frac{6(4+\mu)^{\mu+1}}{4^{4} \Gamma(\mu+4)}, \\
\Lambda & =(4+\mu) / D_{m}, \\
D_{m} & =\frac{\int_{0}^{\infty} N(D) D^{4} d D}{\int_{0}^{\infty} N(D) D^{3} d D}, \\
N_{w} & =\frac{4^{4}}{\pi \rho_{w}}\left(\frac{\mathrm{LWC}}{D_{m}^{4}}\right), \\
\mathrm{LWC} & =\frac{\pi \rho_{w}}{6} \int_{0}^{\infty} N(D) D^{3} d D,
\end{aligned}
$$

$N_{w}\left(\mathrm{~m}^{-3} \mathrm{~mm}^{-1}\right)$ is the normalized intercept of a threeparameter gamma DSD, $\mu$ is the shape parameter, $\rho_{w}$ is the water density $\left(\mathrm{g} \mathrm{cm}^{-3}\right)$, LWC is the liquid water content $\left(\mathrm{g} \mathrm{m}^{-3}\right)$, and $\Gamma$ is the gamma function. From (2), the rain rate can be expressed as

$$
\begin{aligned}
R & =N_{w} I_{R}\left(D_{m}, \mu\right), \\
I_{R} & =6 \pi \times 10^{-4} \int_{0}^{\infty} f(\mu)\left(\frac{D}{D_{m}}\right)^{\mu} \exp (-\Lambda D) D^{3} V(D) d D,
\end{aligned}
$$

where $I_{R}\left(\mathrm{~mm}^{2} \mathrm{~m}^{3} \mathrm{~h}^{-1}\right)$ is the $N_{w}$-normalized rain rate, and $V(D)\left(\mathrm{m} \mathrm{s}^{-1}\right)$ is raindrop fall velocity expressed as a function of $D$. Equating (1) and (3), we arrive at

$$
N_{w}=\frac{\varepsilon^{\tau} a D_{m}^{b}}{I_{R}\left(D_{m}, \mu\right)} .
$$

Also, from (2), the radar reflectivity factor, $Z_{\lambda}$ in $\mathrm{dBZ}$, at a wavelength $\lambda(\mathrm{mm})$ is written as

$$
\begin{aligned}
Z_{\lambda}\left(D_{m}, \mu\right)= & 10 \log _{10}\left(N_{w}\right)+I_{b}\left(D_{m}, \mu, \lambda\right), \\
I_{b}\left(D_{m}, \mu, \lambda\right)= & 10 \log _{10}\left[\frac{\lambda^{4}}{\pi^{5}\left|K_{w}\right|^{2}} \int_{0}^{\infty} f(\mu)\left(\frac{D}{D_{m}}\right)^{\mu}\right. \\
& \left.\times \exp (-\Lambda D) \sigma_{b}(D, \lambda) d D\right],
\end{aligned}
$$

where $I_{b}$ is the $N_{w}$-normalized radar reflectivity in $\log _{10}\left(\mathrm{~mm}^{7}\right)$, and $\sigma_{b}(D, \lambda)$ is the radar backscattering cross section in $\mathrm{mm}^{2}$. The dielectric factor $K_{w}$ is related to the complex refractive index of water. By convention, $\left|K_{w}\right|^{2}$ is taken to be 0.93 . Substituting (4) into (5a), we obtain

$$
\begin{aligned}
Z_{\lambda}\left(D_{m}, \mu\right)= & 10 \log _{10}\left(\varepsilon^{\tau} a\right)+10 b \log _{10}\left(D_{m}\right) \\
& -10 \log _{10}\left[I_{R}\left(D_{m}, \mu\right)\right]+I_{b}\left(D_{m}, \mu, \lambda\right) .
\end{aligned}
$$

Recalling that $a$ and $b$ are storm-type-dependent constants, Eq. (6) provides a relationship between $Z_{\mathrm{Ku}}$ and $D_{m}$, that is, $Z_{\mathrm{Ku}}-D_{m}$ relation with $\varepsilon$ as a parameter. Figure 1 presents an example showing the $Z_{\mathrm{Ku}}-D_{m}$ relation as $\varepsilon$ takes on several values for stratiform (blue) and convective (red) rain. The results show that $Z_{\mathrm{Ku}}$ and $D_{m}$ are related in a one-to-one fashion when $\varepsilon$ is given so that $D_{m}$ can be uniquely derived from $Z_{\mathrm{Ku}}$ using (6). Parameter $N_{w}$ is then obtained from $D_{m}$ and $Z_{\mathrm{Ku}}$ from (5a). Once $N_{w}$ and $D_{m}$ are determined, assuming a fixed $\mu$ of 3 , the specific attenuations at both $\mathrm{Ku}$ and $\mathrm{Ka}$ bands, $k_{\lambda}$ in $\mathrm{dB} \mathrm{km}^{-1}(\lambda=\mathrm{Ku}$ or $\mathrm{Ka})$, are computed by

$$
\begin{aligned}
k_{\lambda}\left(D_{m}, \mu\right)= & N_{w} I_{e}\left(D_{m}, \mu, \lambda\right), \\
I_{e}\left(D_{m}, \mu, \lambda\right)= & 4.343 \times 10^{-3} \int_{0}^{\infty} f(\mu)\left(\frac{D}{D_{m}}\right)^{\mu} \\
& \times \exp (-\Lambda D) \sigma_{e}(D, \lambda) d D,
\end{aligned}
$$




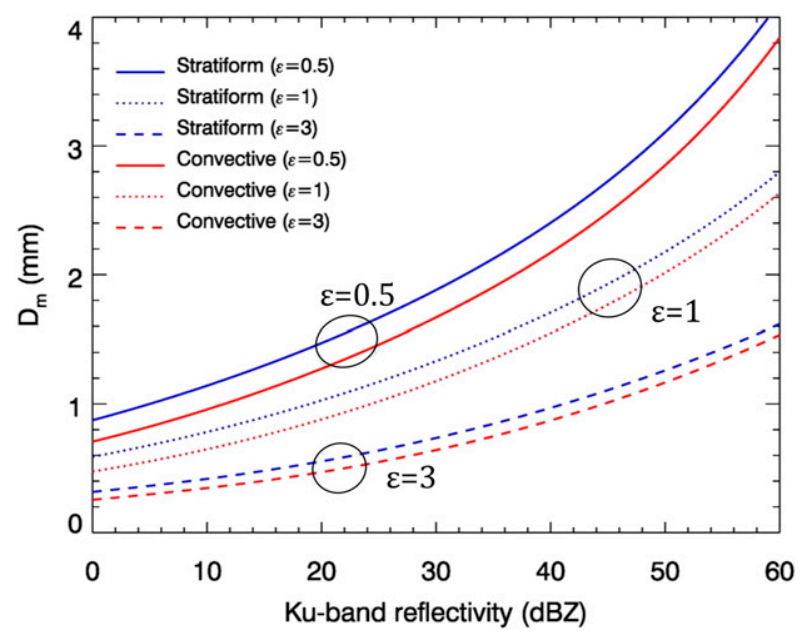

FIG. 1. $D_{m}-Z_{\mathrm{Ku}}$ relations as calculated from (6) for 3 values of $\varepsilon$ of 0.5 (solid lines), 1 (dotted lines), and 3 (dashed lines) as $R-D_{m}$ relations are expressed in (1) for the cases of stratiform (blue) and convective (red) storms.

where $I_{e}$ is the $N_{w}$-normalized specific attenuation in $\mathrm{mm}^{3} \mathrm{~km}$, and $\sigma_{e}(D, \lambda)$ is the extinction cross section in $\mathrm{mm}^{2}$.

Above description comprises the basis of the DPR dual- and single-wavelength retrievals. If $\varepsilon$ is known, gate-by-gate recursive procedures either in the forward direction (from the radar toward the surface) or backward direction can be applied to derive DSD parameters and rainfall rate. An optimization method outlined by Seto et al. (2016) for determination of $\varepsilon$ will be discussed in subsequent sections. Note that a fixed $\mu$ of 3 is assumed for the DPR algorithms (Seto et al. 2013; Seto and Iguchi 2015), and the same assumption $(\mu=3)$ is made throughout the paper.

\section{a. Determination of $\varepsilon$ for dual-wavelength measurements}

The DPR version-5 algorithm uses the forward recursive approach in which the gate-by-gate retrieval proceeds along the radar beam from the storm top to the surface. The best $\varepsilon$ is chosen among a series of $\varepsilon$ test values in such a way that minimizes the predefined cost function. In detail, the procedure is described as follows: Starting with $K$ values of $\varepsilon$ that are equally spaced in $\log _{10}\left(\varepsilon_{k}\right)$ space $(k=1,2, \ldots, K)$, then for each $\varepsilon_{k}$ there correspond unique $R-D_{m}$ and $Z_{\mathrm{Ku}}-D_{m}$ relations given by (1) and (6). For the forward approach, retrieval of the DSD and $R$ profiles is done for each $\varepsilon_{k}$ starting at the top gate and accumulating the attenuation as the retrieval advances toward the surface. The $D_{m}$ is first derived from attenuation-corrected $Z_{\mathrm{Ku}}$ using the $Z_{\mathrm{Ku}}-D_{m}$ relation that corresponds to $\varepsilon_{k} ; N_{w}$ follows from $D_{m}$ and $Z_{\mathrm{Ku}}$. This gate-by-gate retrieval process continues until reaching the final gate which, in practice, is that gate nearest the surface that is uncorrupted by surface clutter. From the estimated DSD profile, the apparent Ka-band reflectivity profile (the reflectivity profile that does not correct rain attenuation, equivalent to the reflectivities being measured) and associated $\delta$ PIA can be simulated. This procedure is repeated for the $K$ values of $\varepsilon$ so that profiles of DSD and $R$ as well as associated $\delta$ PIA and $Z_{\mathrm{Ka}}$ profiles are obtained for each. The final $\varepsilon_{f}$ is chosen as that which minimizes the cost function $p$ that is the sum of $p_{1}, p_{2}$, and $p_{3}$ given by the following equations:

$$
\begin{aligned}
p\left(\varepsilon_{f}\right) & =\min \left[p_{1}\left(\varepsilon_{k}\right)+p_{2}\left(\varepsilon_{k}\right)+p_{3}\left(\varepsilon_{k}\right)\right], \\
p_{1}\left(\varepsilon_{k}\right) & =\frac{\left(\log _{10} \varepsilon_{k}\right)^{2}}{\sigma_{1}^{2}}, \\
p_{2}\left(\varepsilon_{k}\right) & =\frac{\left[\delta \operatorname{PIA}\left(\varepsilon_{k}\right)-\delta \mathrm{PIA}_{\mathrm{SRT}}\right]^{2}}{\sigma_{2}^{2}}, \\
p_{3}\left(\varepsilon_{k}\right) & =\frac{1}{\sigma_{3}^{2}} \sum_{n=1}^{N}\left[Z_{m, n, \text { est }}^{(\mathrm{Ka})}\left(\varepsilon_{k}\right)-Z_{m, n, \mathrm{obs}}^{(\mathrm{Ka})}\right]^{2},
\end{aligned}
$$

where $N$ is the number of range gates along the column; $\delta \operatorname{PIA}\left(\varepsilon_{k}\right)$ is the simulated differential PIA, defined as differences of PIA ( $\mathrm{dB}$ ) between $\mathrm{Ka}$ and $\mathrm{Ku}$ band, as $\varepsilon$ in (1) is set to $\varepsilon_{k} ; \delta$ PIA ${ }_{\mathrm{SRT}}$ is the differential PIA estimated by the SRT (Meneghini et al. 1983); and $Z_{m, n \text {,est }}^{(\mathrm{Ka})}\left(\varepsilon_{k}\right)$ and $Z_{m, n, \text { obs }}^{(\mathrm{Ka})}$ are the simulated and measured Ka-band radar reflectivities in $\mathrm{dB} Z$ at the $n$th range gate, respectively. Parameters $\sigma_{1}, \sigma_{2}$, and $\sigma_{3}$ are the weighting factors: $\sigma_{1}$ is chosen to be the standard deviation of the distribution of $\log _{10}(\varepsilon)$ obtained from the measured DSD data, $\left(\sigma_{2}\right)^{2}$ is the error variance associated with the SRT estimate of $\delta$ PIA, while $\sigma_{3}$ is determined by running simulation tests that yield the best overall retrieval accuracy. There is an apparent advantage of choosing $\delta$ PIA over PIA in (8c) in light of the fact that the error standard deviation of $\delta$ PIA is much smaller than that of the PIA estimated by the SRT. This is because of strong correlation of the normalized surface cross section $\left(\sigma^{0}\right)$ between $\mathrm{Ku}$ and $\mathrm{Ka}$ band (Meneghini et al. 2015). The final solutions of the $D_{m}$ and $R$ profiles are those that correspond to the selected $\varepsilon_{f}$. The approach described above to minimize the cost function is similar to what has been documented by Seto et al. (2016).

To illustrate the detailed step-by-step procedure, a flowchart is provided in Fig. 2, in which $k_{\mathrm{Ku}}$ and $k_{\mathrm{Ka}}$ are specific attenuations in $\mathrm{dB} \mathrm{km}{ }^{-1}$ at $\mathrm{Ku}$ and $\mathrm{Ka}$ band, respectively, computed from estimated DSD parameters. Parameters $A_{\mathrm{Ku}}(n)$ and $A_{\mathrm{Ka}}(n)$ are the accumulated attenuations from the storm top (first gate) to the $n$th gate. To minimize the computations, a lookup table 


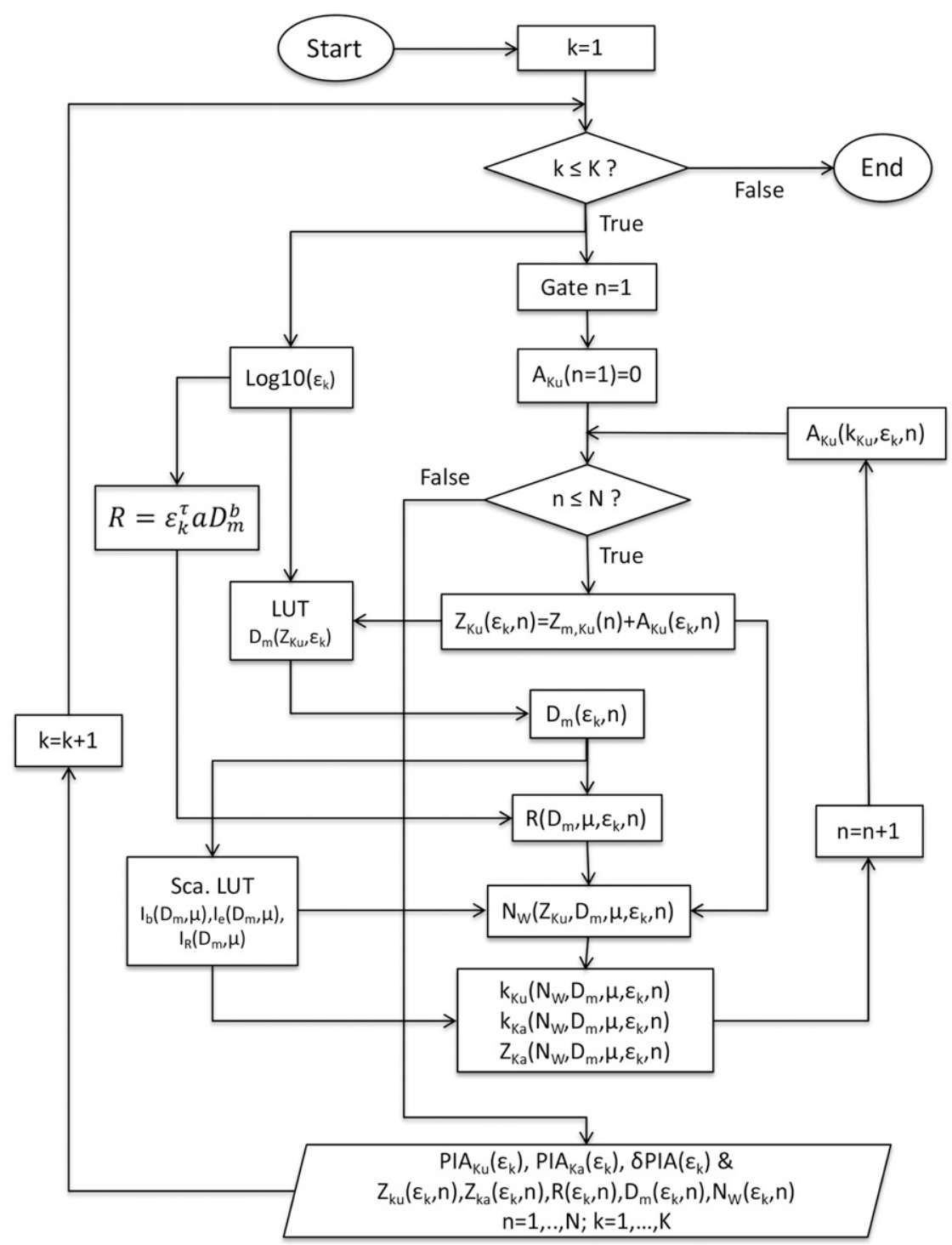

FIG. 2. Flowchart of the DPR algorithms for the retrieval of DSD parameters and rain rate. Outer-loop variable $k$ goes from 1 to $K$ to provide equal-spaced values of $\log _{10}\left(\varepsilon_{k}\right)$ at each step while inner-loop variable $n$, running from 1 to $N$, represents radar range gates at which the retrievals are carried out. A fixed $\mu$ of 3 is assumed in this paper.

(LUT) was created that relates $Z_{\mathrm{Ku}}$ and $D_{m}$ with $\varepsilon$ as parameter at $\mu=3$. For similar reasons the $N_{w}$-normalized integral parameters $I_{R}, I_{b}$, and $I_{e}$, defined in (3), (5), and (7), respectively, were precomputed and stored in a scattering lookup table.

\section{b. Determination of $\varepsilon$ for single-wavelength measurements}

To maintain some degree of retrieval consistency when the retrieval transitions from the dual-wavelength (inner swath of the DPR observation) to the singlewavelength (DPR outer swath), the single-wavelength algorithm functions in the same fashion as the dualwavelength. While the same principles are applied to both the single- and dual-wavelength algorithm, the differences arise from a different set of constraints. The constraints for the single-wavelength approach are given by Seto et al. (2016), which are similar to the following equations:

$$
\begin{aligned}
p\left(\varepsilon_{f}\right) & =\min \left[p_{1}\left(\varepsilon_{k}\right)+p_{2}\left(\varepsilon_{k}\right)\right], \\
p_{1}\left(\varepsilon_{k}\right) & =\frac{\left(\log _{10} \varepsilon_{k}\right)^{2}}{\sigma_{1}^{2}},
\end{aligned}
$$




$$
p_{2}\left(\varepsilon_{k}\right)=\frac{\left[\operatorname{PIA}\left(\varepsilon_{k}\right)-\mathrm{PIA}_{\mathrm{SRT}}\right]^{2}}{\sigma_{2}^{2}} .
$$

The way of finding the best $\varepsilon$ is similar to what has been described for the dual-wavelength, that is, the final $\varepsilon$ or $\varepsilon_{f}$ is chosen to minimize the sum of $p_{1}$ and $p_{2}$. Note that function $p_{1}$ in $(9 \mathrm{~b})$ is identical to that in $(8 \mathrm{~b})$. Instead of $\delta$ PIA being used in $(8 \mathrm{c})$, the single-wavelength PIA is used in $(9 \mathrm{c})$.

The dual-wavelength has one additional constraint resulting from measurements at the second wavelength and a more stringent constraint as a result of the fact that $\delta$ PIA is known to a better accuracy than the singlewavelength PIA. These differences constitute an advantage of the dual-wavelength over the single-wavelength retrieval.

\section{c. Backward recursive approach}

As indicated earlier, the DPR-operational algorithm uses the forward recursive approach in which the retrieval starts from storm top, and then proceeds downward into storm until reaching the gate closest to the surface free from surface clutter. In contrast, the backward recursion begins at the clutter-free gate closest to the surface, and then moves upward until reaching the storm top. For the backward approach, the PIA estimates are needed to correct the measured radar reflectivities at the gate nearest the surface to obtain attenuation-corrected reflectivities. Although the PIA is most often estimated by the SRT, the procedure would be the same if any other method, such as a radiometer-derived estimate, were used. For dualfrequency implementation, this means that the path attenuation must be estimated at both wavelengths or that the path attenuations must be derived from $\delta$ PIA, both procedures of which incur larger errors than the use of the $\delta$ PIA constraint employed in the forward recursion. Although the retrieval principles remain the same, the retrieval accuracy of the forward and backward approaches is affected by different error sources. The pros and cons of these two approaches will be discussed later in the paper. It is necessary to reiterate that the DPR-operational algorithm adopts the forward approach, and the backward approach described above is merely an interesting alternative.

\section{Evaluation of DPR algorithms}

The DPR single- and dual-wavelength profiling retrieval algorithms are assessed by using realistic hydrometeor profiles obtained from measured DSD data acquired from a variety of storm systems during NASA field campaigns. From the measured spectra, parameters associated with both radar and hydrometeors, which include true and measured radar reflectivities, attenuation, rain rate and DSD characteristic parameters $\left(D_{m}\right.$ and $N_{w}$ ), are directly computed. For computation of radar-related scattering cross sections, such as $\sigma_{b}$ in (5b) and $\sigma_{e}(7 \mathrm{~b})$, the raindrops are prescribed to be oblate spheroids with axial ratios that follow the shape-size relations reported by Thurai et al. (2007). The symmetry axes of the raindrops are aligned in the vertical direction, and nadir-viewing radar is assumed. The T-matrix method is used to compute the scattering properties of single particles (Mishchenko and Travis 1998). The rain and DSD parameters are computed according to the equations given in (2) and (3). The simulated $\mathrm{Ku}$ - and Ka-band apparent reflectivity profiles are then used as inputs to the retrieval algorithms. The degree to which the radar estimates agree with the true values, which are also derived from the DSD-simulated profiles, is used as a measure of retrieval accuracy and as a means to evaluate the impact of different model assumptions and constraints used in the retrievals. The DSD data used in this study are primarily from the measurements made by the Parsivel disdrometers during the Iowa Flood Studies (IFloodS) field experiment from 1 May to 15 June 2013, the Integrated Precipitation Validation Experiment (IPHEx) from 1 May to 15 June 2014, and the observations at the NASA Wallops Flight Facility in Wallops Island, Virginia, from 2013 to 2014. This collection constitutes a total of approximately 170000 one-minute rain DSD spectra across three different sites and time periods, $80 \%$ of which result in reflectivities that exceed the DPR sensitivity thresholds of 12 and $16 \mathrm{dBZ}$ for $\mathrm{Ku}$ and $\mathrm{Ka}$ band, respectively. As our focus is on the DPR algorithms, only those DSD data are used that lead to the $\mathrm{Ku}-$ and Ka-band reflectivities greater than their respective thresholds.

To examine the algorithms for different storm systems, vertical hydrometeor profiles are constructed from measured DSDs with varying degrees of vertical DSD correlation. Two types of vertical DSD profile are particularly important: one with a vertically uniform DSD where a single DSD size spectrum is used for entire column; the second is the vertically nonuniform profile in which a time series of DSD spectra are converted to a vertical profile by successively assigning 1-min-integrationtime DSD data to radar range gates from the storm top to the surface. The former is the simplest case while the latter approximates observations in nature based on the fact that vertically mean correlations of rain rate, $D_{m}$ and $\log _{10} N_{w}$ obtained from such profiles generally conform to the results from a combined study of Micro Rain Radar (MRR) and DSD measurements reported by Adirosi et al. (2016). To mimic the DPR observation, 
the vertical range resolution is set to $0.125 \mathrm{~km}$ with a rain-layer thickness of $5 \mathrm{~km}$.

Our evaluation is focused primarily on the estimates of rain rate and $D_{m}$ with a particular emphasis on the DPR dual-wavelength algorithms not only because they are the DPR core products but also serve as reference for other instruments. Although there are many factors that might affect the algorithm performance, our evaluation study is carried out on the performance-related issues that include: 1) comparisons of the forward and backward recursive algorithms; 2) impact of nonuniformity of hydrometeor profiles on algorithm performance; 3 ) sensitivity of the DPR estimates to the initial $R-D_{m}$ relations that are chosen; 4) analysis of the roles of individual constraints to the retrieval solution; 5) retrieval uncertainties caused by $\delta$ PIA errors; 6) comparison of single- and dualwavelength performance.

\section{a. Forward and backward approaches}

To perform the retrieval, the nominal $R-D_{m}$ relation is chosen as "stratiform" rain when the path-averaged rain rate is less than $5 \mathrm{~mm} \mathrm{~h}^{-1}$ and "convective" otherwise. Figure 3 provides comparisons between the estimated and true $D_{m}$ when the DPR dual-wavelength algorithm (forward approach) is applied to nonuniform DSD profiles. Two-dimensional probability density functions (PDF) computed from the estimated and true data points are shown in the left column with the color scales representing data distribution while the means (thick blue lines) and twice the standard deviations (vertical bars) are plotted on the right. To track the changes in retrieval accuracy along the profiles, the comparisons are made at range gates at the rain top (top panel) and at the near-surface (bottom panel) range that correspond to the beginning and end of the profile. An unbiased $\delta$ PIA model with the standard deviation of $0.8 \mathrm{~dB}$ is used to characterize the PIA errors. This surface model is consistent with the GPM DPR observations over ocean (Meneghini et al. 2015). Comparisons of rain rate from the same DSD dataset are shown in Fig. 4. Careful examination of these results reveals that $D_{m}$ and rain rates derived from the DPR are generally in fairly good agreement with the true values despite the small biases in rain rate that occurs at light-to-moderate rain rates. The error variance of the estimated $D_{m}$ increases slightly with an increase of $D_{m}$ while the relative error variance in rain rate marginally decreases with an increase in rain rate. Retrieval errors are basically independent of height.

With the same DSD profiles, the estimates obtained from the backward approach are shown in Fig. 5 where the PDF of $D_{m}$ is given in the left panel and $R$ in right panel. In addition to the $\delta$ PIA error model needed, the PIAs at $\mathrm{Ku}$ and $\mathrm{Ka}$ band are required to compensate for the attenuations in the measured reflectivities at the near-surface gate. Like $\delta$ PIA, the PIA error is approximated by an unbiased Gaussian distribution with standard deviation of $2 \mathrm{~dB}$, which is close to those of the DPR measurements at nadir over the ocean according to the findings reported by Meneghini et al. (2015). Comparisons of the results from the forward (Figs. 3, 4) and backward (Fig. 5) approaches show comparable results where both produce reasonably fairly accurate estimates of $D_{m}$ and rain rate.

The error sources that affect the forward and backward retrievals are not exactly the same. For example, the errors associated with attenuation correction due to cloud water and melting layer above the rain affect the performance of the forward approach but not that of the backward approach. These uncertainties, however, are not considered in this study. Another difference is the need in the backward formulation to use the single-frequency attenuations to correct the near-surface measured reflectivities, adding errors to the retrieval.

\section{b. Nonuniformity of hydrometeor profiles}

As noted earlier, the nonuniform profiles generated by using time series DSD data give a reasonably accurate statistical representation of the vertical variations in the DSD that occur in nature. To explore how the retrieval performance changes as the degree of nonuniformity changes, we test the retrievals on two extreme profiles: fully correlated or uniform DSD and uncorrelated DSD in which vertical DSD profiles are formed by randomly choosing a DSD spectrum out of the DSD data pool at each range gate of the profiles. The fully correlated or uniform profile approximates the case of stratiform rain while the latter results in extremely nonuniform profiles that provides an upper bound to the DSD variability along the profile. Uniform profiles, from the perspective of the retrieval algorithms, present the best case (with the least uncertainties) because it conforms to one of the important assumptions of the algorithm that employs only a single adjustment factor $(\varepsilon)$. Conversely, the extremely nonuniform profiles are expected to be associated with the largest retrieval errors in the sense that a single $\varepsilon$ is unable to account for the DSD changes.

Figures 6 and 7 show comparisons of $D_{m}$ and $R$, respectively, between the estimated and true values for the cases of fully correlated (left column), partially correlated (center column) and uncorrelated DSD profiles. Note that for reference the results of $D_{m}$ and $R$ shown in Figs. 3 and 4 are included in Figs. 6 and 7, respectively, to 

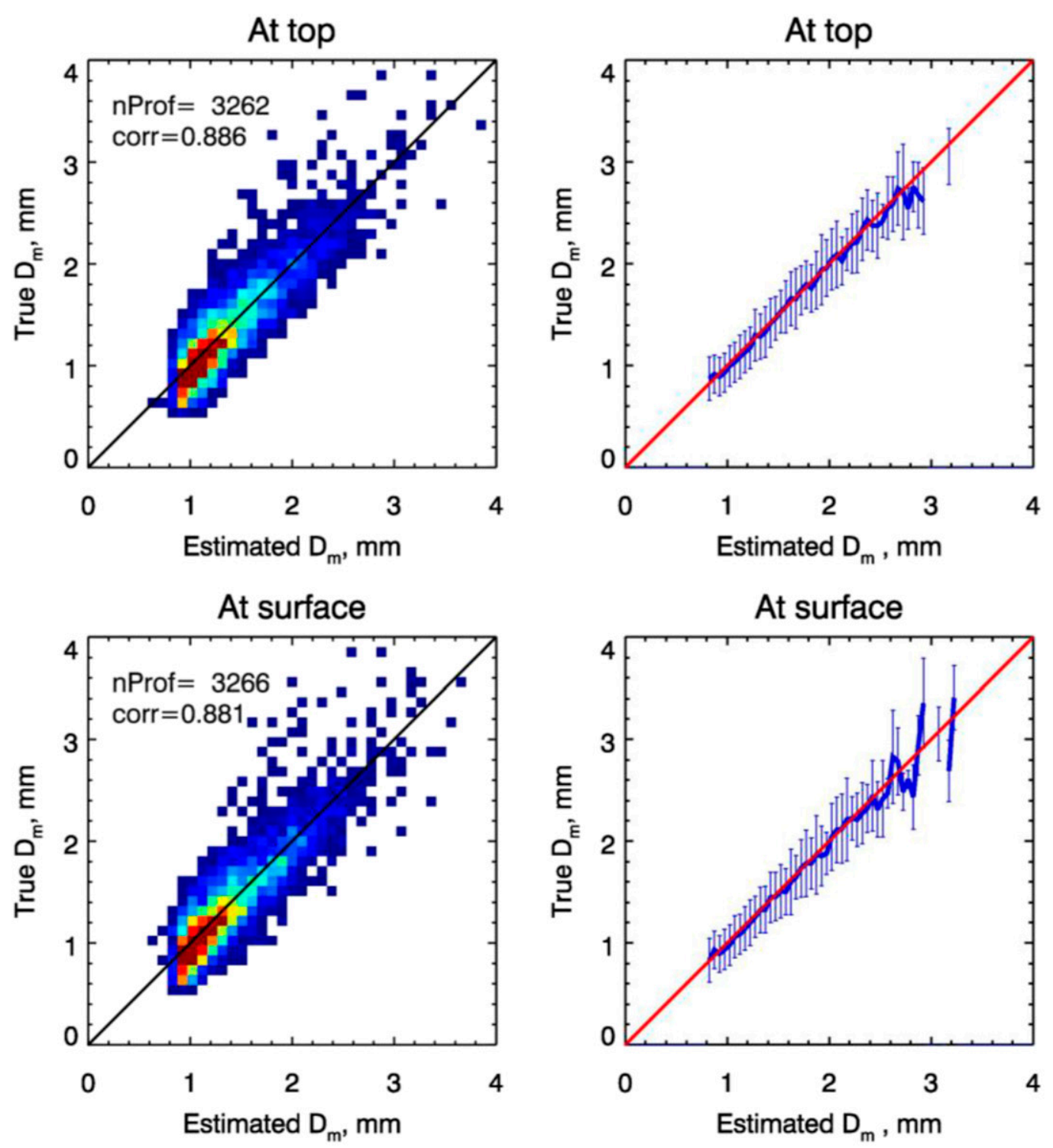

PDF

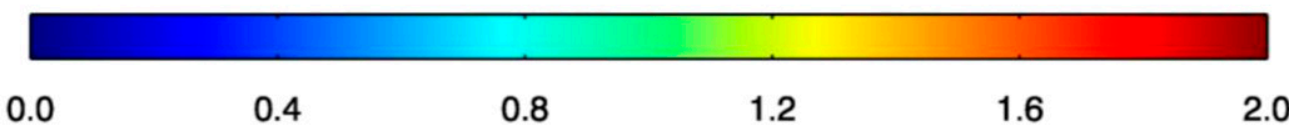

FIG. 3. Comparisons of $D_{m}$ estimated by the DPR dual-wavelength algorithm with the true $D_{m}$ at the gates of rain (top) top and (bottom) surface as the forward recursive approaches are applied to nonuniform DSD profiles. (left) Two-dimensional probability density functions (PDFs) obtained from the data points of estimated and true $D_{m}$, and (right) the means (thick blue curves) and the two-time standard deviations (thin blue vertical bars) derived from the same data points. An unbiased statistical $\delta$ PIA model with the standard deviation of $0.8 \mathrm{~dB}$ is assumed. One-to-one relations (black and red lines in the left and right panels, respectively) are also plotted for references.

represent the case of partially correlated DSD profile. As before, the comparisons are shown only at rain-top and near-surface gates. The results show that uncertainties in the $D_{m}$ estimates, represented by twice the error standard deviation (thin vertical bars), increase with changes of the DSD profiles from uniform, partially correlated and then to fully uncorrelated. Uncertainties in the rain rate estimates begin as very small for the cases 

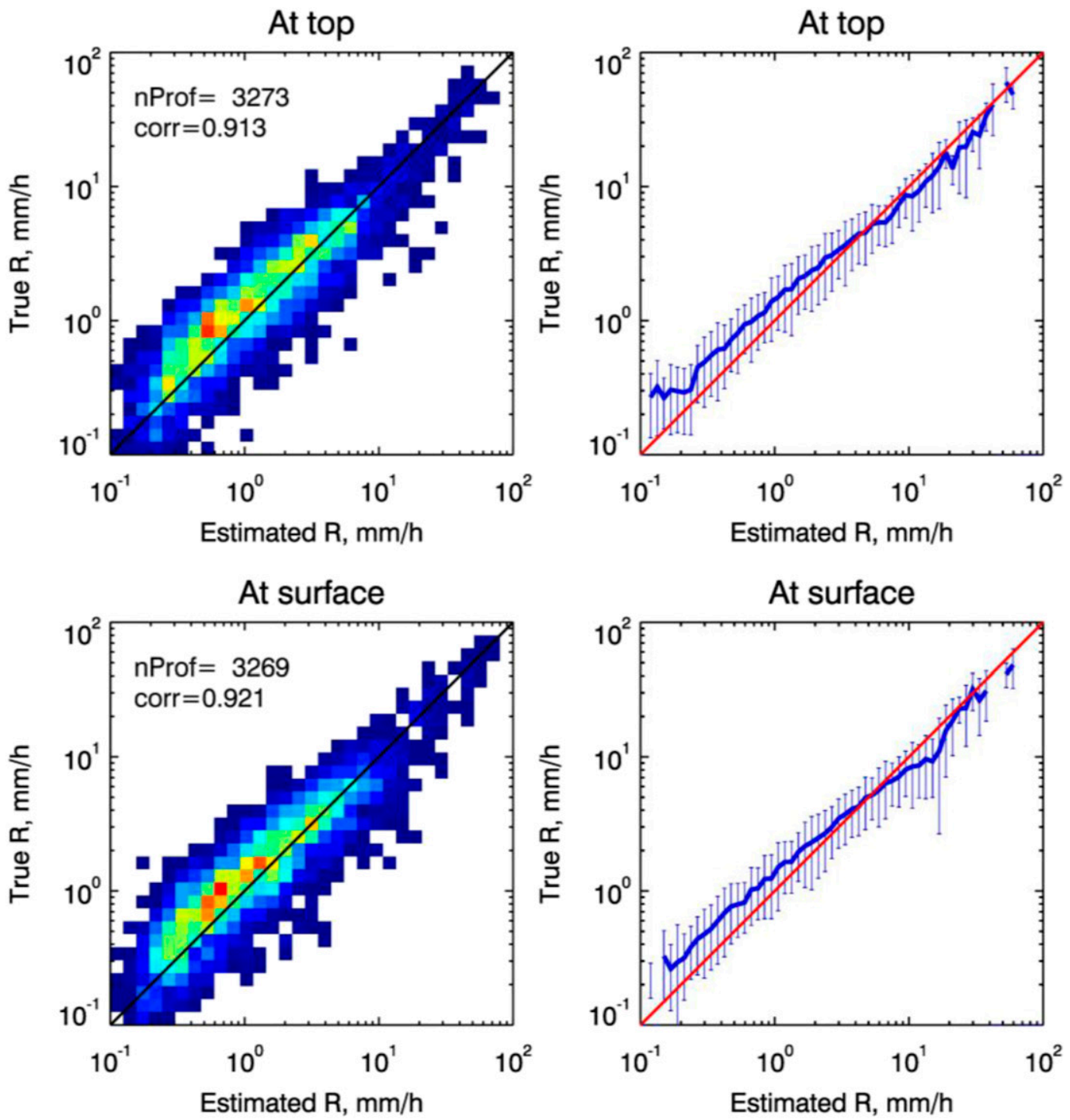

PDF

of uniform profiles when rain is greater than $5 \mathrm{~mm} \mathrm{~h}^{-1}$ and grow larger as the DSD nonuniformity increases.

\section{c. Impact of $R-D_{m}$ relations}

The $R-D_{m}$ relation is one of the important components in the operational DPR algorithm. Analysis of measured DSD data shows large variations in the relationship between $R$ and $D_{m}$. Clearly, a constant $R-D_{m}$ relation cannot account for the space-time variations in the DSD. Adjustment of the $\varepsilon$ parameter in the $R-D_{m}$ relation attempts to mitigate the uncertainties resulting from the DSD variations. The DPR employs one $R-D_{m}$ relation for stratiform rain and another for convective rain, both of which are applied globally. 

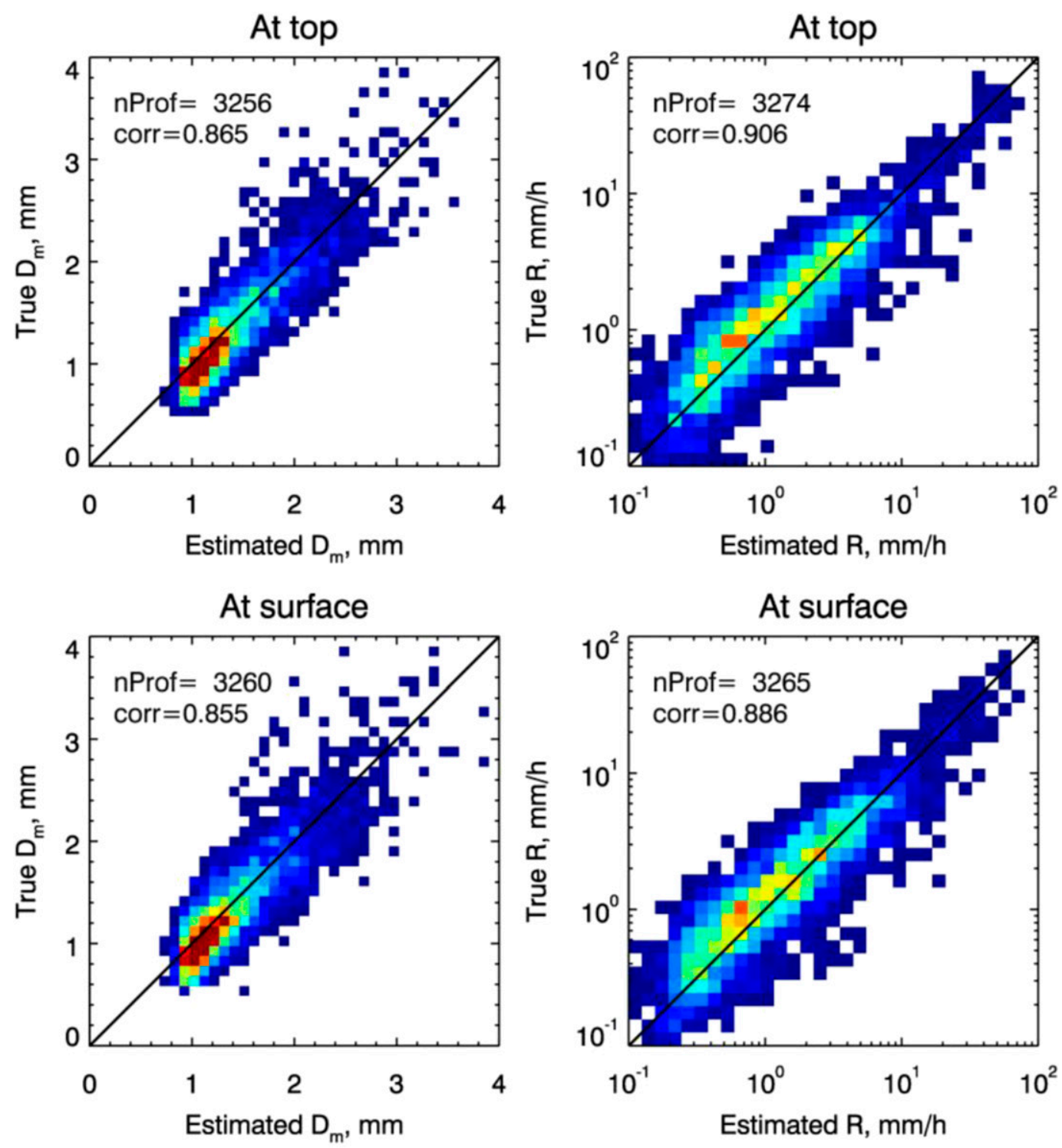

PDF

\section{0}

0.4

0.8

1.2

1.6

FIG. 5. PDFs of (left) $D_{m}$ and (right) $R$ estimated by the DPR dual-wavelength algorithm with the true rain rates at the gates of rain (top) top and (bottom) surface as the backward recursive approaches are applied to nonuniform DSD profiles. An unbiased statistical $\delta$ PIA model with the standard deviation of $0.8 \mathrm{~dB}$ is assumed. One-to-one relations (black lines) are also plotted for references.

In the study that follows, we will test how the estimates are sensitive to the choice of the default $R-D_{m}$ relations. To do this, the DPR-default $R-D_{m}$ relations are replaced by those derived from aforementioned DSD dataset, and the derived $D_{m}$ and $R$ using different $R-D_{m}$ relations are compared with those using the DPR-default $R-D_{m}$ relations.
The regressions of $R$ and $D_{m}$ are made using the DSD data described previously, and their fitting parameters, defined in (1), are summarized in Table 1. For reference, the coefficients of the $R-D_{m}$ relations for the DPRoperational algorithms are also included in the table. Two separate DSD datasets, which correspond to the 

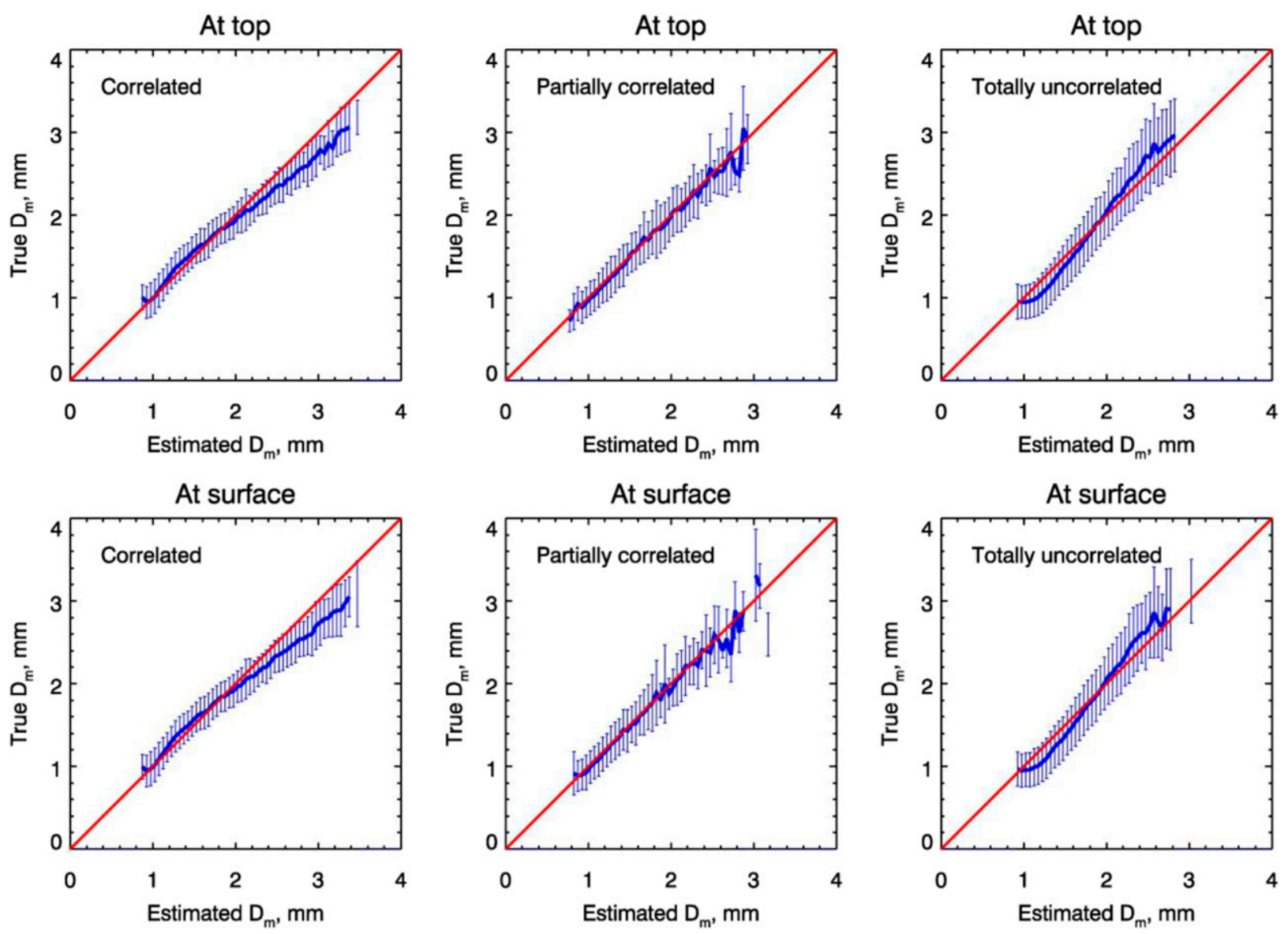

FIG. 6. Comparisons of $D_{m}$ estimated by the DPR dual-wavelength algorithm with true $D_{m}$ at the gates of rain (top) top and (bottom) surface as the forward recursive approaches are applied to the fully correlated (uniform), partially correlated (nonuniform), and totally uncorrelated (extremely nonuniform) vertical DSD profiles. The means (thick blue curves) and the two-time square root of error variances (thin blue vertical bars) are computed from the data points of estimated and true $D_{m}$ within intervals between $D_{m}$ and $D_{m}+\Delta D_{m}$, where $\Delta D_{m}$ is $0.1 \mathrm{~mm}$. An unbiased statistical $\delta$ PIA model with the standard deviation of $0.8 \mathrm{~dB}$ is assumed. One-to-one relations (red lines) are also plotted for references.

measurements from the Parsivel and 2DVD disdrometers, are employed. Unlike the nominal DPR relations that are broken into the categories of stratiform and convective storms, only one $R-D_{m}$ relation is obtained across entire DSD data. As seen clearly from the table, the coefficients of the $R-D_{m}$ relations between the DPR-default and DSD-derived results differ to some extent. The differences between the Parsivel and 2DVD datasets are, however, quite small because they are largely deployed in the same places. Although the Parsivel and 2DVD disdrometers have different sensitivities in measuring small and large drops, agreement between these two instruments indicate the careful work performed by the teams that conducted the ground campaigns and quality-checked the data.

Accuracies of the retrieved $D_{m}$ and $R$ are depicted in Figs. 8 and 9, respectively, with use of the DPR (left columns), Parsivel-derived (middle columns), and
2DVD-derived (right columns) $R-D_{m}$ relations as the nonuniform vertical DSD profiles are assumed. It is obvious that the results of $D_{m}$ and $R$ from these three $R-D_{m}$ relations are comparable despite small biases found in rain estimates from the DPR $R-D_{m}$ relation. Agreement of these results suggests that estimates of $D_{m}$ and $R$ are not highly sensitive to the choice of initial $R-D_{m}$ relations. Insensitivity of the retrievals to initial $R-D_{m}$ relations implies convergence of the solutions, which, in practice, is a significant benefit for the retrieval in view of highly variable $R$ and $D_{m}$ relationship.

\section{d. Roles of individual constraints to retrieval solutions}

As discussed earlier, finding the correct adjustment factor, $\varepsilon_{f}$, is crucial to achieve accurate estimates of $D_{m}$ and $R$. The constraints expressed in (8) play an important role in the search for $\varepsilon_{f}$. Three independent constraint functions $\left(p_{1}, p_{2}\right.$, and $\left.p_{3}\right)$ characterizing different 

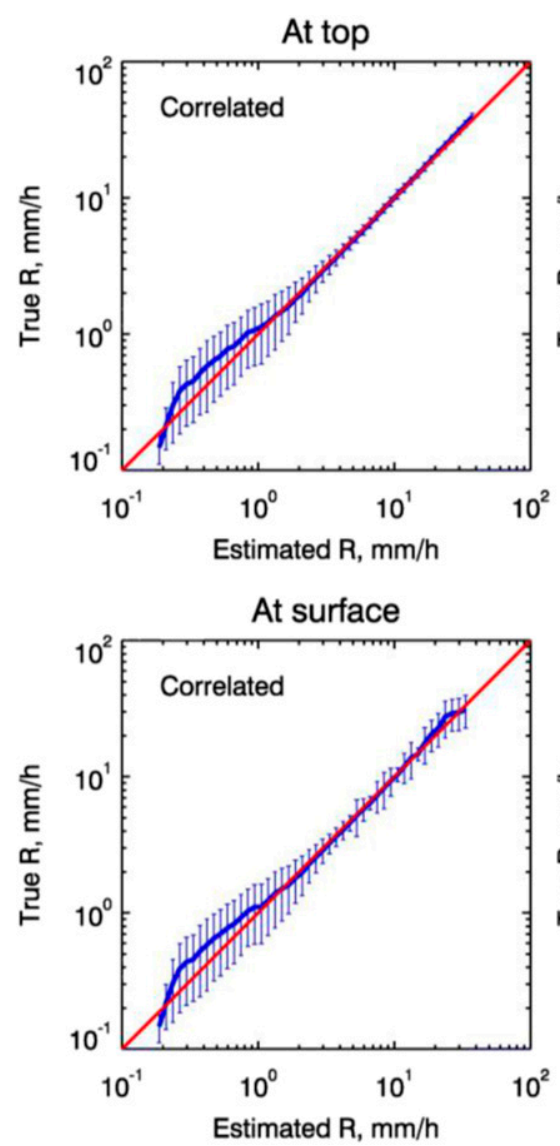
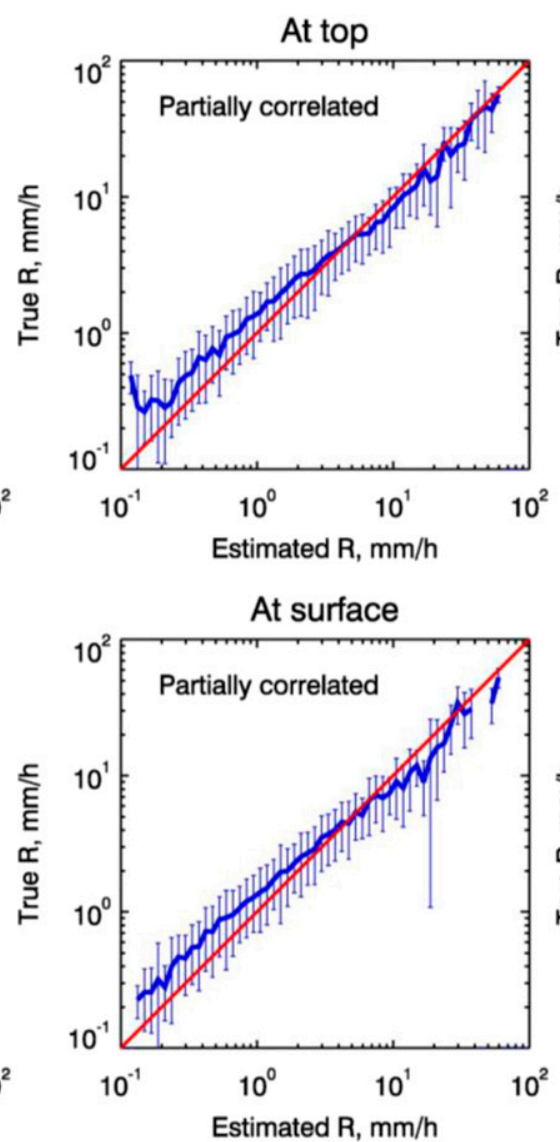
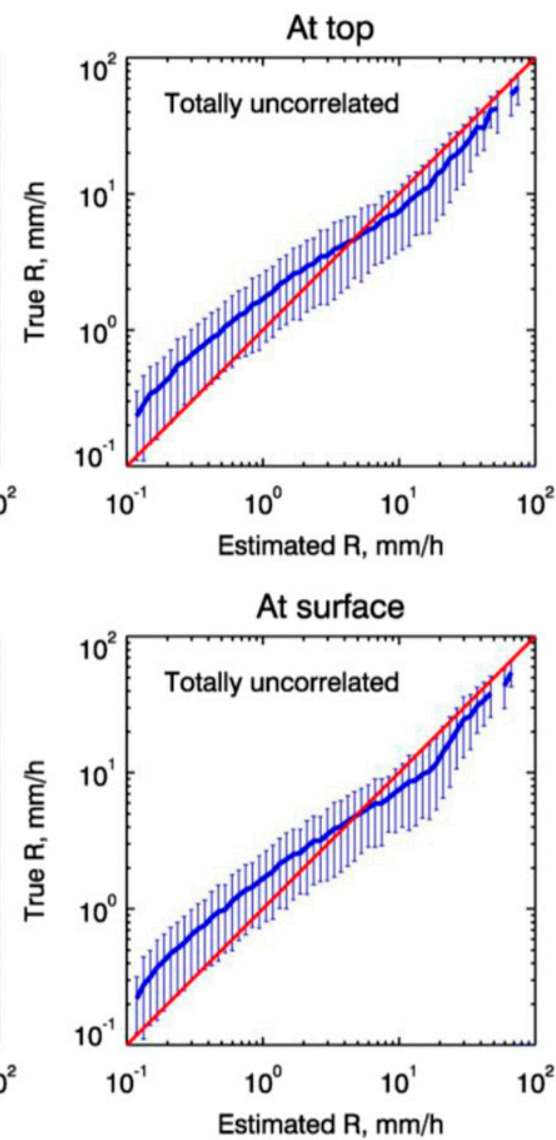

FIG. 7. Comparisons of rain rates estimated by the DPR dual-wavelength algorithm with their truth at the gates of rain (top) top and (bottom) surface as the forward recursive approaches are applied to the fully correlated (uniform), partially correlated (nonuniform), and totally uncorrelated (extremely nonuniform) vertical DSD profiles. The means (thick blue curves) and the two-time square roots of error variances (thin blue vertical bars) are computed from the data points of estimated and true $R$ within intervals between $\log _{10} R$ and $\log _{10} R+$ $\Delta \log _{10} R$, where $\Delta \log _{10} R$ is 0.1 and $R$ is in $\mathrm{mm} \mathrm{h}^{-1}$. An unbiased statistical $\delta$ PIA model with the standard deviation of $0.8 \mathrm{~dB}$ is assumed. One-to-one relations (red lines) are also plotted for references.

radar and hydrometeor properties tend to constrain the solutions in different ways. Because of this, the strength of a particular constraint is not constant but depends on rain intensity. To examine the respective roles of the $p_{1}$, $p_{2}$, and $p_{3}$ constraints, we run the algorithm using only a single constraint at a time.

The left column of Fig. 10 shows comparisons between the estimated rain rates and their true values in terms of the two-dimensional PDF with $p_{1}$ or (8b) being used alone as a constraint. Similarly, as shown in the middle and right columns of Fig. 10 rain rates are retrieved using only constraint $p_{2}$ (middle) or $p_{3}$ (right). The results indicate that $p_{1}$ provides fairly a strong constraint over the entire range of rain rates while $p_{2}$ strongly constrains rain estimates for heavy rain but is weak at light rain rates. Parameter $p_{3}$ provides a fairly strong constraint at light rain and even stronger for heavy rain. The level of the relative constraint strengths of $p_{1}, p_{2}$, and $p_{3}$ are summarized in Table 2 . These results imply that better estimates might be achieved if $p_{2}$ and $p_{3}$ are weighted more heavily for the moderate-to-heavy rain while $p_{1}$ and $p_{3}$ are weighted more for light rain. Note that the above discussion on the constraint of $p_{2}$ is based on the PIA error model from the DPR observation over the ocean where the standard deviation of $\delta$ PIA is around $0.8 \mathrm{~dB}$. This would change, however, if surface conditions or uncertainties of $\delta$ PIA were altered. This will be discussed in the subsequent section.

TABLE 1. Coefficients of the $R-D_{m}$ relations expressed in (1) that are derived from the DSD data collected by the Parsivel and 2DVD disdrometers during several NASA-sponsored field campaigns. For reference, coefficients used for the DPR algorithms are also included.

\begin{tabular}{lccc}
\hline \hline & $a$ & $b$ & $\tau$ \\
\hline Parsivel & 0.469 & 3.002 & 2.403 \\
2DVD & 0.470 & 3.002 & 2.564 \\
DPR stratiform & 0.401 & 6.131 & 4.649 \\
DPR convective & 1.370 & 5.420 & 4.258 \\
\hline
\end{tabular}



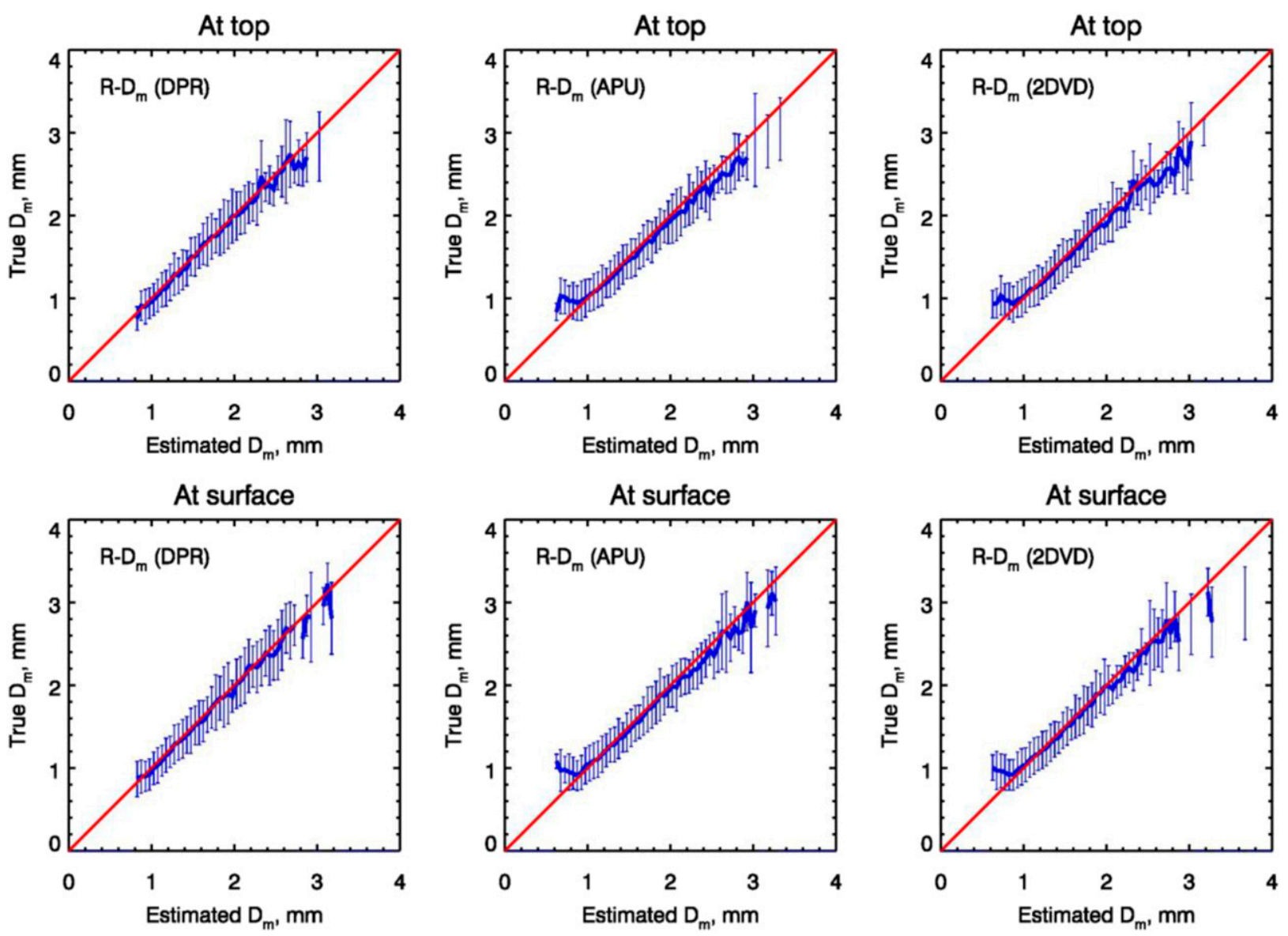

FIG. 8. Comparisons of $D_{m}$ estimated by the DPR dual-wavelength algorithms with different $R-D_{m}$ relations being used with true $D_{m}$ at the gates of rain (top) top and (bottom) surface as the forward recursive approaches are applied to the nonuniform vertical DSD profiles. The results from (left) DPR-default, (middle) Parsivel-DSD-derived, and (right) 2DVD-DSD-derived $R-D_{m}$ relations are shown. The means (thick blue curves) and the two-time square root of error variances (thin blue vertical bars) are computed from the data points of estimated and true $D_{m}$ within intervals between $D_{m}$ and $D_{m}+\Delta D_{m}$, where $\Delta D_{m}$ is $0.1 \mathrm{~mm}$. An unbiased statistical $\delta$ PIA model with the standard deviation of $0.8 \mathrm{~dB}$ is assumed. One-to-one relations (red lines) are also plotted for references.

\section{e. Retrieval uncertainties caused by $\delta P I A$ errors}

The weighting factor $\sigma_{2}$ in (8) is related to the error in the $\delta$ PIA estimate, which in turn depends on the variability of the frequency difference of the surface cross sections. As an unbiased $\delta$ PIA model is assumed, Fig. 11 shows the comparisons of the estimated rain rates when three $\delta$ PIA error standard deviations of 0.4 (left column), 1.2 (middle column), and $2 \mathrm{~dB}$ (right column) are assumed to represent the cases of small, moderate and large uncertainties of $\delta$ PIA, respectively. For these comparisons, the dual-wavelength retrievals are applied to the nonuniform DSD profiles. The results show that the accuracy of the rain estimates degrades more severely for light rain than for heavy rain as the $\delta$ PIA uncertainties increase. This behavior occurs because a fixed amount of error in the $\delta$ PIA error has a relatively large impact on light rain retrievals and conversely. It implies that the $\delta$ PIA-related constraint generally works better for heavy rain than it does for light rain. In other words, a reduction in the $\delta$ PIA uncertainty, though leading to an overall improvement of the retrieval, is more beneficial at light rain rates than at heavy rain rates.

\section{f. Comparison of single- and dual-wavelength performance}

As described in section 2, the single- and dual-wavelength algorithms share the same methodology in estimating $D_{m}$ and $R$; the difference lies in the constraints that are used to find the best $\varepsilon$. Having modeled the PIA and $\delta$ PIA as unbiased Gaussian distributions with standard deviations of 2 and $0.8 \mathrm{~dB}$, respectively, the $D_{m}$ and rain rates estimated by the single $\mathrm{Ku}$ - and $\mathrm{Ka}$-band (middle and right columns, respectively) as well as 

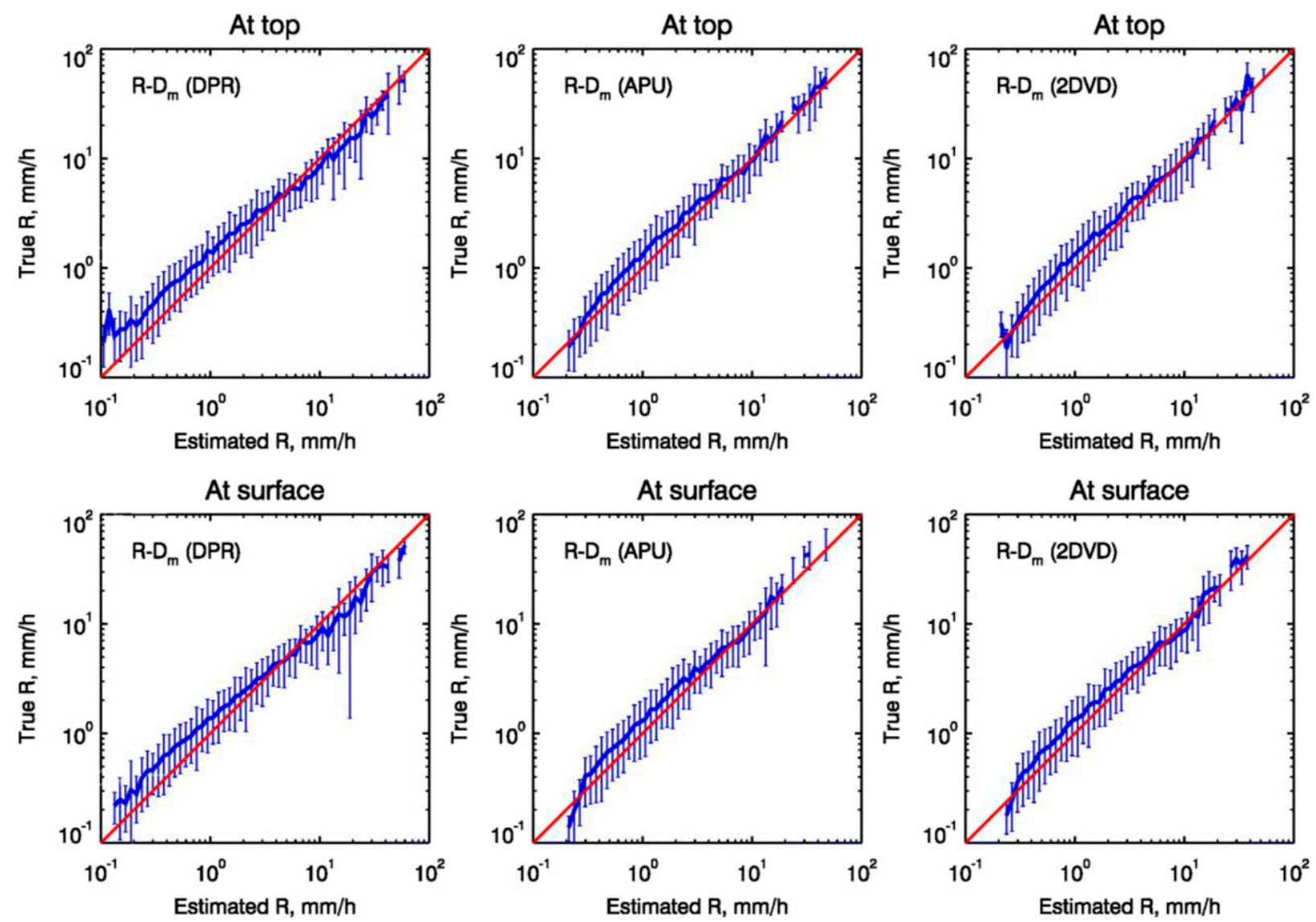

FIG. 9. Comparisons of rain rates estimated by the DPR dual-wavelength algorithms with different $R-D_{m}$ relations being used with true $D_{m}$ at the gates of rain (top) top and (bottom) surface as the forward recursive approaches are applied to the nonuniform vertical DSD profiles. The results from (left) DPR-default, (middle) Parsivel-DSD-derived, and (right) 2DVD-DSD-derived $R-D_{m}$ relations are shown. The means (thick blue curves) and the two-time square root of error variances (thin blue vertical bars) are computed from the data points of estimated and true $R$ within intervals between $\log _{10} R$ and $\log _{10} R+\Delta \log _{10} R$, where $\Delta \log _{10} R$ is 0.1 and $R$ is in mm h An unbiased statistical $\delta$ PIA model with the standard deviation of $0.8 \mathrm{~dB}$ is assumed. One-to-one relations (red lines) are also plotted for references.

dual-wavelength (left column) algorithms are shown in Figs. 12 and 13 for a range gate at the rain top (top row) and near-surface (bottom row). For these results, the nonuniform DSD profiles are assumed. As anticipated, the dual-wavelength algorithms consistently provide more accurate estimates of $D_{m}$ and rain rate than either of the $\mathrm{Ku}$ - and $\mathrm{Ka}$-band single-wavelength retrievals. For relatively large $D_{m}$, the estimates of $D_{m}$ from the single-wavelength not only show a higher degree of uncertainty but also larger biases than the dualwavelength. The Ka-band rain retrieval exhibits excellent agreement with truth for moderate-to-heavy rains if attenuation is either absent (storm top) or perfectly corrected. It, however, deteriorates rapidly as the retrieval proceeds downward toward the surface as a result of errors associated with the attenuation correction. Uncertainties in the Ku-band rain retrievals, on the other hand, show insignificant differences, excepting heavy rain cases, between the beginning and end of the profiles, which is a result of the relatively small attenuation at this frequency.

\section{Summary and remarks}

To assess performance of current GPM DPR algorithms and to gain insight into ways to improve the retrievals, a physically accurate and realistic evaluation of the methods is required. To achieve this, measured DSD data acquired from a variety of storm systems during several NASA field campaigns are employed to construct hydrometeor profiles. The measured DSD spectra are used to compute parameters of the radar and rain, which include true and measured radar reflectivities, attenuation, rain and characteristics of the DSD. These parameters of rain are taken as truth while the simulated measured reflectivities serve as inputs to the retrieval algorithms. 

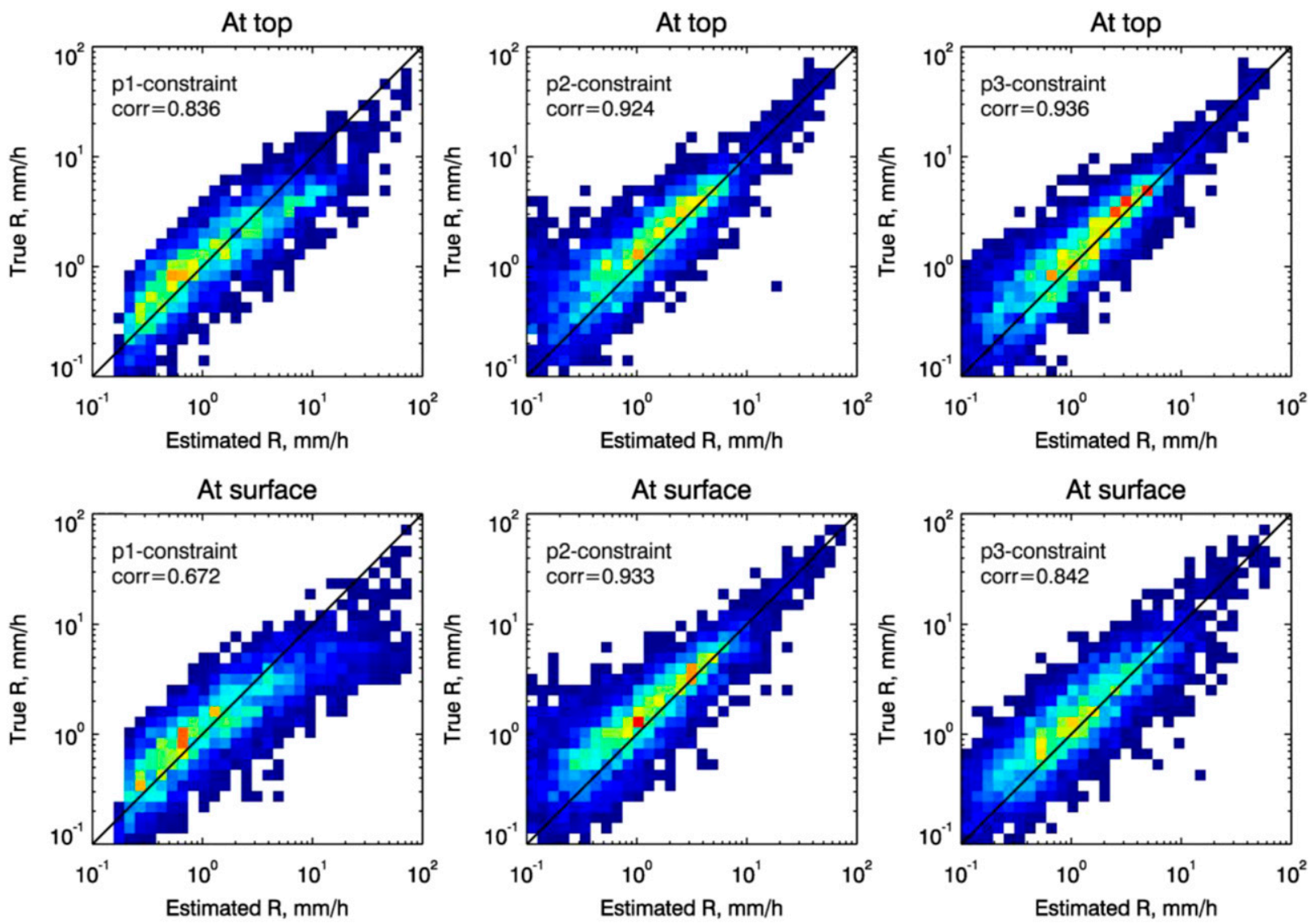

PDF

$$
0.0
$$

0.4

0.8

1.2

1.6

2.0

FIG. 10. PDFs of rain rates estimated by the DPR dual-wavelength algorithms and true rain rates as the algorithms employ only single individual constraints in selection of $\varepsilon$, such as (left) $p_{1}$, (middle) $p_{2}$, and (right) $p_{3}$ expressed in (8b), (8c), and (8d), respectively. Comparisons are made at the gates of rain (top) top and (bottom) surface as the forward recursive approaches are applied to nonuniform DSD profiles. An unbiased statistical $\delta$ PIA model with the standard deviation of $0.8 \mathrm{~dB}$ is assumed. One-to-one relations (black lines) are also plotted for references.

The DPR algorithms adopt an optimal approach. To obtain consistent estimates across the single- and dualwavelength measurements, the same principles are applied for both approaches but with different constraints. Modifying the $R-D_{m}$ relation through adjustment of $\varepsilon$ allows the retrievals to account for DSD variations from profile to profile. Finding the best $\varepsilon$ is a crucial procedure that directly affects retrieval accuracy of $D_{m}$ and $R$. For the dual-wavelength retrieval, three constraint functions of $p_{1}, p_{2}$, and $p_{3}$ in (8) are used in the search

TABLE 2. Strength levels of individual constraints $\left(p_{1}, p_{2}\right.$, and $\left.p_{3}\right)$ to the retrieval solutions and remarks on their respective errors. Constraint strengths are divided into 4 levels from the weakest to strongest: weak, fair, strong, and very strong.

\begin{tabular}{lllll}
\hline \multicolumn{1}{c}{$R\left(\mathrm{~mm} \mathrm{~h}^{-1}\right)$} & $R<1$ & $1<R<10$ & $R>10$ & Remarks \\
\hline$p_{1}(\varepsilon)=\frac{\left(\log _{10} \varepsilon\right)^{2}}{\sigma_{1}^{2}}$ & Fair & Fair & Fair & Possible bias \\
$p_{2}(\varepsilon)=\frac{\left[\delta \mathrm{PIA}(\varepsilon)-\delta \mathrm{PIA}_{\mathrm{SRT}}\right]^{2}}{\sigma_{2}^{2}}$ & Weak & Fair/strong & Very strong & Depends on $\delta$ PIA errors \\
$p_{3}(\varepsilon)=\frac{1}{\sigma_{3}^{2}} \sum_{n+1}^{N}\left[Z_{m, n, \text { est }}^{(\mathrm{Ka})}(\varepsilon)-Z_{m, n, \mathrm{obs}}^{(\mathrm{Ka})}\right]^{2}$ & Fair & Fair/strong & Very strong & Possible bias when $R<1$ \\
\hline
\end{tabular}



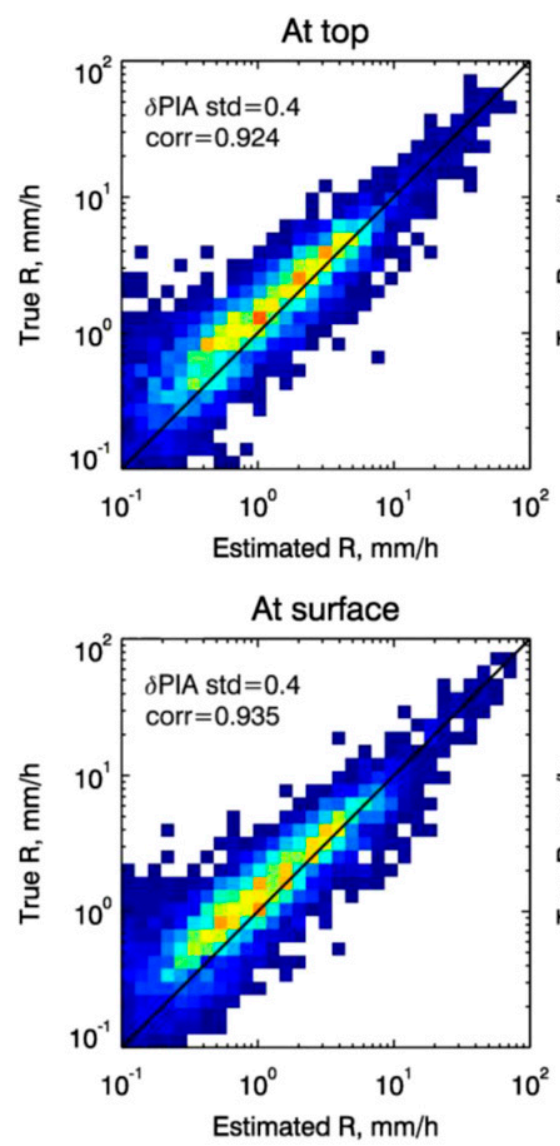

Estimated $\mathrm{R}, \mathrm{mm} / \mathrm{h}$
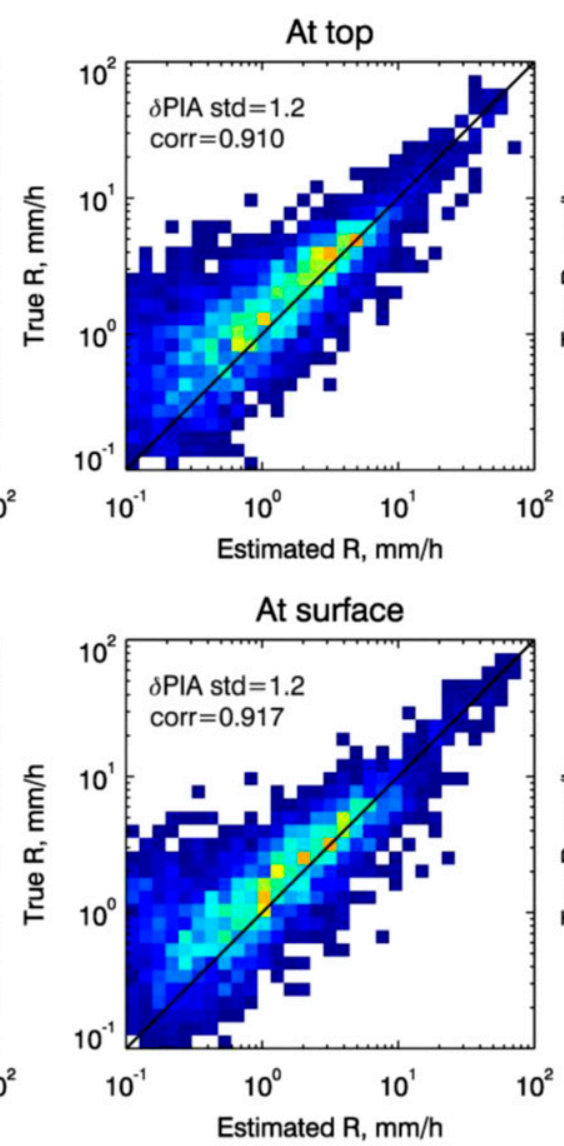
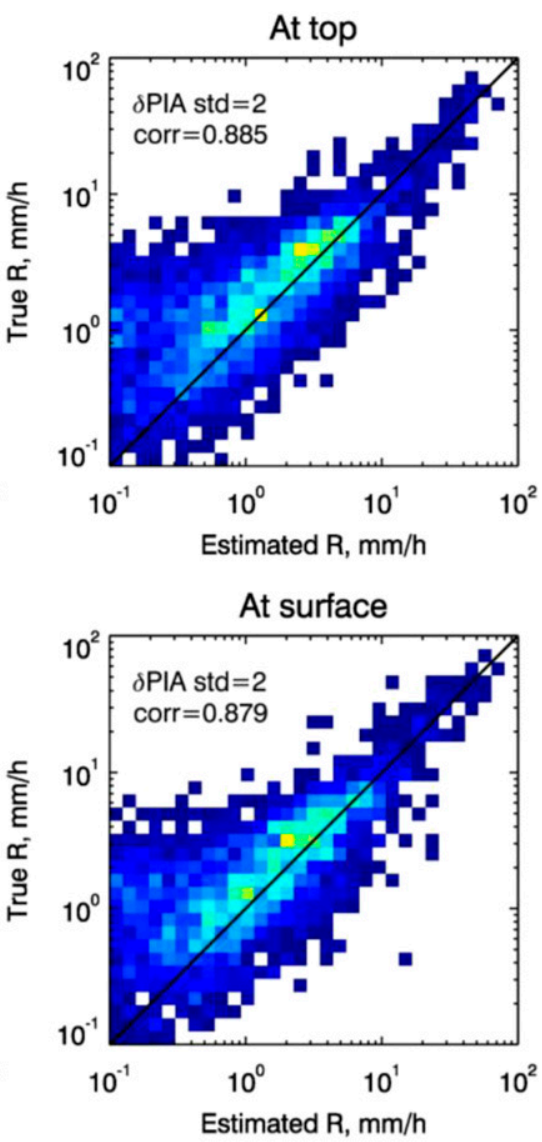

PDF

FIG. 11. PDFs of rain rates estimated by the DPR dual-wavelength algorithms and true rain rates at the gates of rain (top) top and (bottom) surface at several $\delta$ PIA error variances as the forward recursive approaches are applied to nonuniform DSD profiles. An unbiased statistical $\delta$ PIA model with the standard deviations of (left) $0.4 \mathrm{~dB}$, (middle) $1.2 \mathrm{~dB}$, and (right) $2 \mathrm{~dB}$ is assumed. One-to-one relations (black lines) are also plotted for references.

for $\varepsilon$, whereas two constraints of $p_{1}$ and $p_{2}$ given in (9) are used for the single-wavelength algorithm. Of these constraint functions, $p_{1}$, charactering the $\varepsilon$ distribution that is usually derived from DSD spectra, is shared by both the single- and dual-wavelength procedures. Constraint $p_{2}$, defined in (8) and (9), are similar in form but the former uses the differential path attenuation while the latter uses the single-frequency path attenuation. Constraint $p_{3}$ relates to the difference between the simulated and measured Ka-band measured reflectivity profiles, and is clearly applicable only to the dualwavelength solution.

The nonuniform DSD profiles, constructed by using 1-min time series DSD spectra to represent the vertical column, are primarily used for the evaluations because they approximately resemble realistic profiles based on the fact that spatial correlations of these profiles are in reasonable agreement with those found from the studies reported by Adirosi et al. (2016) and Liao and Meneghini (2019). In addition to hydrometeor profiles for the retrieval evaluation, error models characterizing PIA and $\delta$ PIA uncertainties are needed. They follow unbiased Gaussian distributions with standard deviations similar to the DPR observations over the ocean. The algorithms are also applied to a couple of extreme cases, such as fully correlated and uncorrelated DSD profiles, to test robustness of the algorithms and to estimate the upper error bound. The impact of the $R-D_{m}$ relation chosen for the retrieval is examined by comparing the DPR estimates obtained by using the $R-D_{m}$ relations extracted from different DSD datasets. Effect of the PIA and $\delta$ PIA estimate errors on the retrievals is 

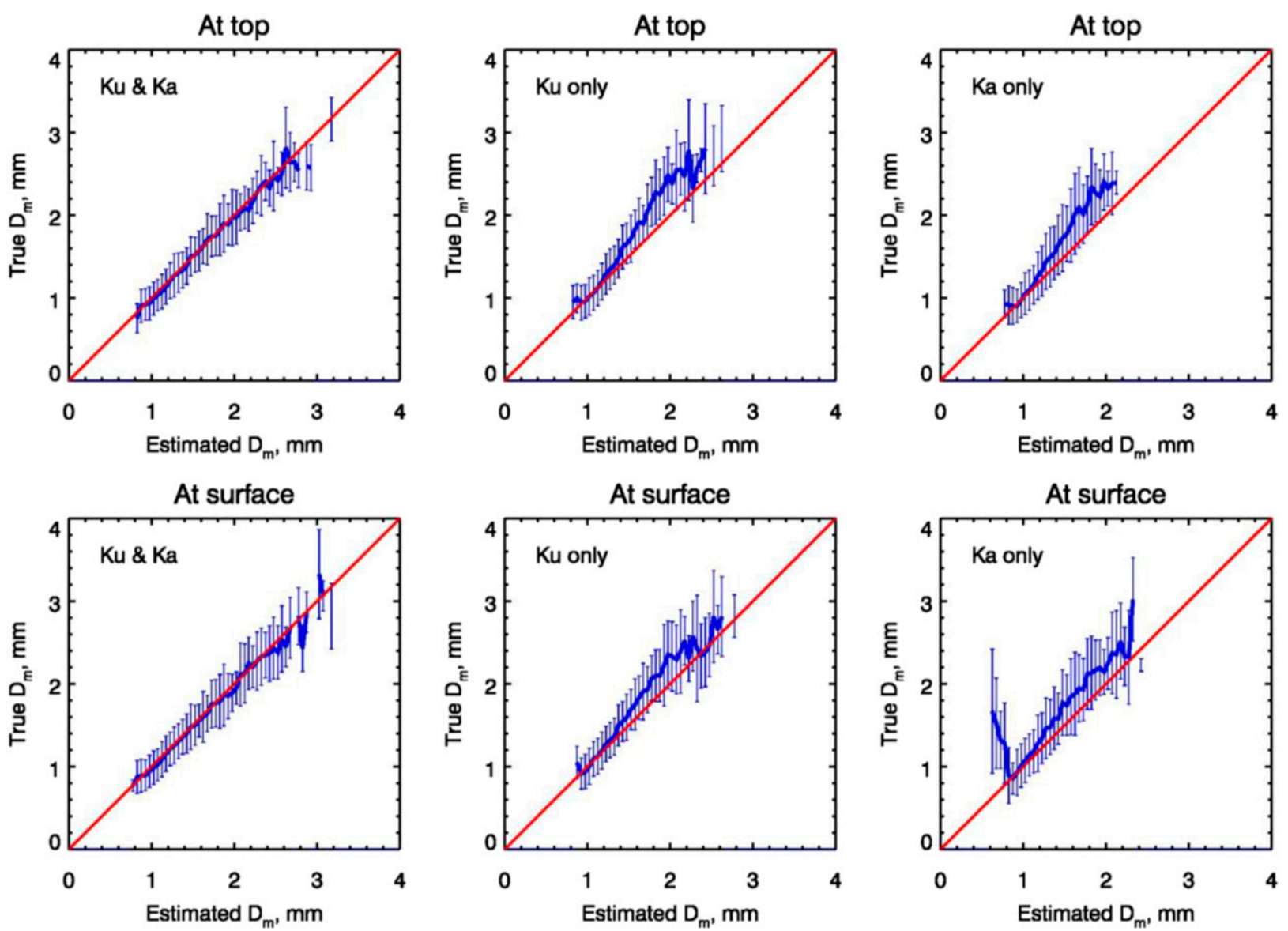

FIG. 12. Comparisons of the DPR (left) dual-wavelength, and (middle),(right) single-wavelength (for Ku and Ka band, respectively) performances in estimating $D_{m}$ at the gates of rain (top) top and (bottom) surface as the DPR-like forward recursive approaches are applied to the nonuniform vertical DSD profiles. The means (thick blue curves) and the two-time square root of error variances (thin blue vertical bars) are computed from the data points of estimated and true $D_{m}$ within intervals between $D_{m}$ and $D_{m}+\Delta D_{m}$, where $\Delta D_{m}$ is $0.1 \mathrm{~mm}$. An unbiased statistical $\delta$ PIA model with the standard deviation of $0.8 \mathrm{~dB}$ is assumed. One-to-one relations (red lines) are also plotted for references.

assessed through comparisons of the retrieval results by varying the magnitudes of the uncertainties. Investigation into strengths and weaknesses of individual constraints $\left(p_{1}, p_{2}\right.$, and $\left.p_{3}\right)$ to the retrieval solutions is carried out to gain the knowledge of how the weighting factors influence the retrieval accuracy. Comparisons of the retrieval accuracy of the DPR single- and dualwavelength algorithms are conducted to examine the advantages of the second frequency.

Overall, the DPR dual-wavelength algorithms perform fairly well from the storm top to the storm bottom yielding nearly unbiased estimates of $D_{m}$ when they are applied to nonuniform DSD profiles. Biases and error standard deviations of the estimated $D_{m}$ are generally well below $0.5 \mathrm{~mm}$ over the full range of $D_{m}$. This is also true for rain estimates that show strong correlations with their true values despite slight underestimations for light rain. In addition to the forward approach
(DPR-operational algorithm), also evaluated is the backward approach that uses the same principle as the forward approach. The results from the backward approach suggest that the retrieval accuracy is comparable to the forward approach.

Comparisons of the retrieval results from the fully correlated to totally uncorrelated profiles show that the assumption of single $\varepsilon$ along the vertical profile leads to an increase in error in the estimation of $D_{m}$ as the degree of decorrelation increases. This increase of error is even more significant in the estimation of $R$. Because of the $\varepsilon$ adjustment factor, the retrievals of $D_{m}$ and $R$ are not highly sensitive to the initial choice in the $R-D_{m}$ relation so that the retrievals eventually converge to nearly the same solution irrespective of the initial relationship.

Analysis of the impact of individual constraint strengths on the retrieval reveals that the $p_{1}$ constraint 

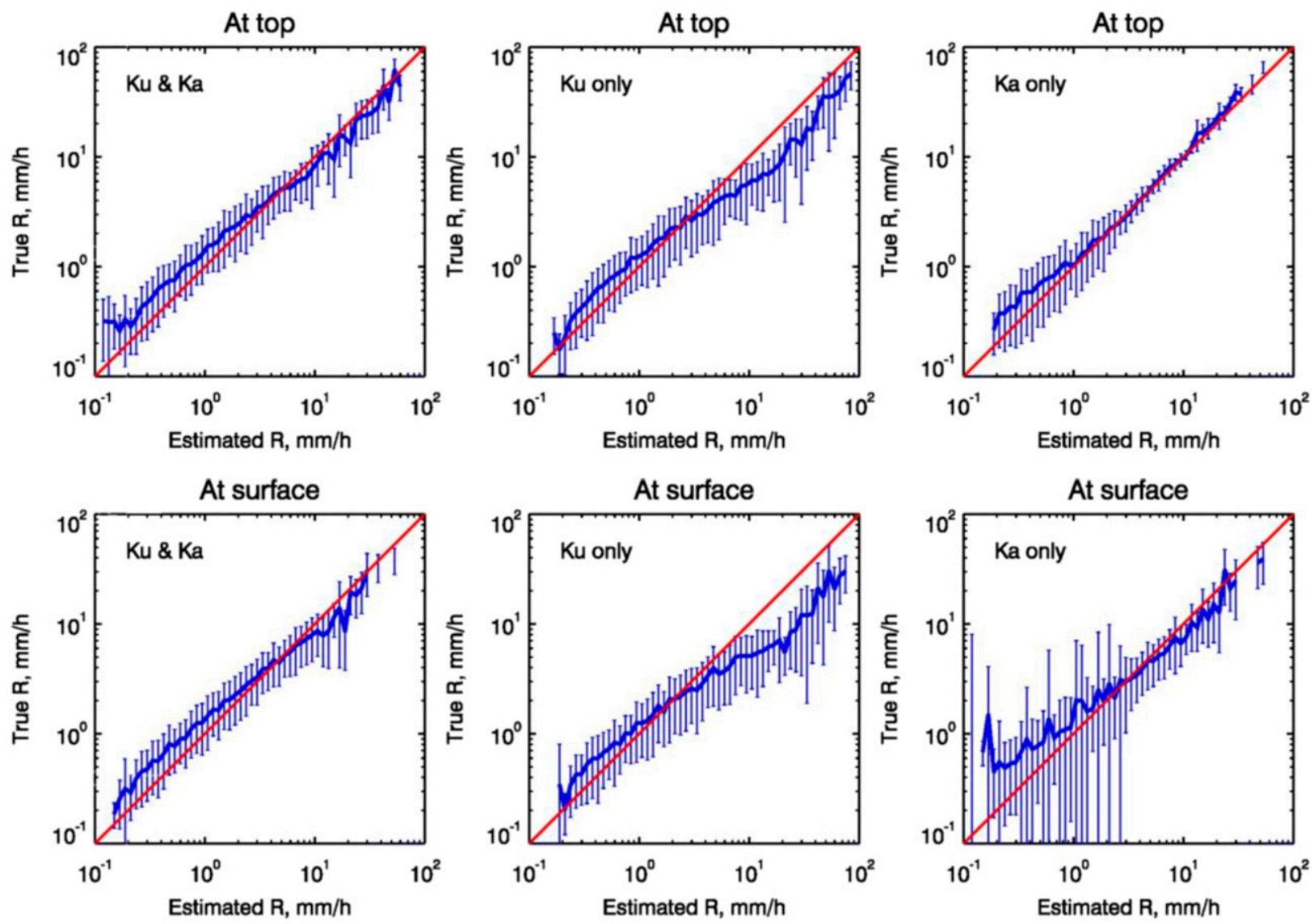

FIG. 13. Comparisons of the DPR (left) dual-wavelength and (middle),(right) single-wavelength (for Ku band and Ka band, respectively) performances in estimating rain rates at the gates of rain (top) top and (bottom) surface as the DPR-like forward recursive approaches are applied to the nonuniform vertical DSD profiles. The means (thick blue curves) and the two-time square root of error variances (thin blue vertical bars) are computed from the data points of estimated and true $R$ within intervals between $\log _{10} R$ and $\log _{10} R+$ $\Delta \log _{10} R$, where $\Delta \log _{10} R$ is 0.1 and $R$ is in $\mathrm{mm} \mathrm{h}^{-1}$. An unbiased statistical $\delta$ PIA model with the standard deviation of $0.8 \mathrm{~dB}$ is assumed. One-to-one relations (red lines) are also plotted for references.

is significant at all rain rates. The $p_{2}$ and $p_{3}$, on the other hand, provide strong constraints in heavy rain but weak constraints in light rain. An improvement of the retrievals might be achieved if the weighting factors associated with these constraints were made functions of the rain intensity. An error associated with the $\delta$ PIA estimates leads to relatively large uncertainties in rain estimates for light rain but relatively small ambiguities for heavy rain. In other words, an improvement of $\delta$ PIA estimates would significantly help to reduce uncertainties at light rain rates. A hybrid scheme that takes advantage of several independent techniques in estimates of PIA and $\delta$ PIA shows promise in reducing errors, particularly in light rain rates (Meneghini et al. 2018). In general, better accuracy in the PIA and $\delta$ PIA estimates is achieved over the ocean than over the land (Meneghini et al. 2015). The same DPR algorithm is, however, applied across different types of surface (ocean, land, and coast). The PIA/ $\delta$ PIA-estimated accuracy is directly related to the weighting factor $\left(\sigma_{2}\right)$ of the function $p_{2}$. In principle, the algorithm works even without good PIA/ $\delta$ PIA estimates but with degraded retrieval accuracy.

Comparisons of the single- and dual-wavelength retrievals show that the dual-wavelength algorithms provide better estimates of DSD parameters and rain rate than the single-wavelength algorithms consistently throughout the entire profile. This comes as no surprise as the dual-wavelength data provides additional constraints on the solution.

Acknowledgments. This work is supported by Dr. R. Kakar of NASA Headquarters under NASA's Precipitation Measurement Mission (PMM) Grant NNH15ZDA001NPMM. The authors also wish to thank Dr. Ali Tokay from University of Maryland Baltimore County for providing DSD data. 


\section{REFERENCES}

Adirosi, E., L. Baldini, N. Roberto, P. Gatlin, and A. Tokay. 2016: Improvement of vertical profiles of raindrop size distribution from micro rain radar using $2 \mathrm{D}$ video disdrometer measurements. Atmos. Res., 169, 404-415, https://doi.org/10.1016/ j.atmosres.2015.07.002.

Bringi, V. N., and V. Chandrasekar, 2001: Polarimetric Doppler Weather Radar: Principles and Applications. Cambridge University Press, $664 \mathrm{pp}$.

_- G. Huang, V. Chandrasekar, and E. Gorgucci, 2002: A methodology for estimating the parameters of a Gamma raindrop size distribution model from polarimetric radar data: Application to a squall-line event from the TRMM/Brazil campaign. J. Oceanic and Atmos. Tech., 19, 633-645, https:// doi.org/10.1175/1520-0426(2002)019<0633:AMFETP > 2.0. CO;2.

Chandrasekar, V., W. Li, and B. Zafar, 2005: Estimation of raindrop size distribution from spaceborne radar observations. IEEE Trans. Geosci. Remote Sens., 43, 1078-1086, https:// doi.org/10.1109/TGRS.2005.846130.

Feingold, G., and Z. Levin, 1986: The lognormal fit to raindrop spectra from frontal convective clouds in Israel. J. Appl. Meteor., 25, 1346-1363, https://doi.org/10.1175/1520-0450(1986) 025<1346:TLFTRS $>2.0$. CO;2.

Gorgucci, E., G. Scarchilli, V. Chandrasekar, and V. Bringi, 2000: Measurement of mean raindrop shape from polarimetric radar observations. J. Atmos. Sci., 57, 3406-3413, https://doi.org/ 10.1175/1520-0469(2000)057<3406:MOMRSF>2.0.CO;2.

,,,--- and,- 2002 : Estimation of raindrop size distribution parameters from polarimetric radar measurements. J. Atmos. Sci., 59, 2373-2384, https://doi.org/10.1175/15200469(2002)059<2373:EORSDP > 2.0.CO;2.

Grecu, M., L. Tian, W. S. Olson, and S. Tanelli, 2011: A robust dual frequency radar profiling algorithm. J. Appl. Meteor. Climatol., 50, 1543-1557, https://doi.org/10.1175/2011JAMC2655.1.

Hou, A., and Coauthors, 2014: The global precipitation measurement mission. Bull. Amer. Meteor. Soc., 95, 701-722, https:// doi.org/10.1175/BAMS-D-13-00164.1.

Iguchi, T., and R. Meneghini, 1994: Intercomparison of singlefrequency methods for retrieving a vertical rain profile from airborne or spaceborne radar data. J. Atmos. Oceanic Technol., 11, 1507-1516, https://doi.org/10.1175/1520-0426(1994)011<1507: IOSFMF $>2.0 . \mathrm{CO} ; 2$.

_- T. Kozu, R. Meneghini, J. Awaka, and K. Okamoto, 2000: Rain profiling algorithm for the TRMM Precipitation Radar. J. Appl. Meteor., 39, 2038-2052, https://doi.org/10.1175/15200450(2001)040<2038:RPAFTT >2.0.CO;2.

_ - and Coauthors, 2009: Uncertainties in the rain profiling algorithm for the TRMM Precipitation Radar. J. Meteor. Soc. Japan, 87A, 1-30, https://doi.org/10.2151/jmsj.87A.1.

Kozu, T., T. Iguchi, T. Shimomai, and N. Kashiwagi, 2009: Raindrop size distribution modeling from a statistical rain parameter relation and its application to the TRMM precipitation radar rain retrieval algorithm. J. Appl. Meteor. Climatol., 48, 716-724, https://doi.org/10.1175/2008JAMC1998.1.

Liao, L., and R. Meneghini, 2005: A study of air/space-borne dualwavelength radar for estimates of rain profiles. Adv. Atmos. Sci., 22, 841-851, https://doi.org/10.1007/BF02918684.

_, and —_, 2009a: Validation of TRMM Precipitation Radar through Comparison of its multiyear measurements with ground-based radar. J. Atmos. Oceanic Technol., 48, 804-817, https://doi.org/10.1175/2008JAMC1974.1.
- and - 2009b: Changes in the TRMM version-5 and version-6 precipitation radar products due to orbit boost. J. Meteor. Soc. Japan, 87A, 93-107, https://doi.org/10.2151/ jmsj.87A.93.

, and - 2019: A modified dual-wavelength technique for Ku- and Ka-band radar rain retrieval. J. Appl. Meteor. Climatol., 58, 3-18, https://doi.org/10.1175/JAMC-D-18-0037.1.

,-- , and T. Iguchi, 2001: Comparisons of rain rate and reflectivity factor derived from the TRMM precipitation radar and the WSR-88D over the Melbourne, Florida, site. J. Atmos. Oceanic Technol., 18, 1959-1974, https://doi.org/10.1175/15200426(2001)018<1959:CORRAR > 2.0.CO;2.

$\longrightarrow,-$ A. Tokay, and L. F. Bliven, 2016: Retrieval of snow properties for Ku- and Ka-band dual-frequency radar. J. Appl. Meteor. Climatol., 55, 1845-1858, https://doi.org/10.1175/ JAMC-D-15-0355.1.

Mardiana, R., T. Iguchi, and N. Takahashi, 2004: A dual-frequency rain profiling method without the use of a surface reference technique. IEEE Trans. Geosci. Remote Sens., 42, 2214-2225, https://doi.org/10.1109/TGRS.2004.834647.

Meneghini, R., J. Eckerman, and D. Atlas, 1983: Determination of rain rate from a spaceborne radar using measurements of total attenuation. IEEE Trans. Geosci. Remote Sens., GE-21, 34 43, https://doi.org/10.1109/TGRS.1983.350528.

— T. Kozu, H. Kumagai, and W. C. Boncyk, 1992: A study of rain estimation methods from space using dual-wavelength radar measurements at near-nadir incidence over ocean. J. Atmos. Oceanic Technol., 9, 364-382, https://doi.org/10.1175/ 1520-0426(1992)009<0364:ASOREM>2.0.CO;2.

—, H. Kumagai, J. R. Wang, T. Iguchi, and T. Kozu, 1997: Microphysical retrievals over stratiform rain using measurements from an airborne dual-wavelength radar radiometer. IEEE Trans. Geosci. Remote Sens., 35, 487-506, https://doi.org/10.1109/ 36.581956 .

, H. Kim, L. Liao, J. A. Jones, and J. M. Kwiatkowski, 2015: An initial assessment of the Surface Reference Technique applied to data from the Dual-Frequency Precipitation Radar (DPR) on the GPM Satellite. J. Atmos. Oceanic Technol., 32, 22812296, https://doi.org/10.1175/JTECH-D-15-0044.1.

, L. Liao, T. Iguchi, and H. Kim, 2018: Hybrid estimates of path attenuation for the DPR. 2018 IEEE International Geoscience and Remote Sensing Symposium, Valencia, Spain, IEEE, 8327-8330, https://doi.org/10.1109/IGARSS.2018.8518017.

Mishchenko, M. I., and L. D. Travis, 1998: Capabilities and limitations of a current FORTRAN implementation of the Tmatrix method for randomly oriented, rotation symmetric scatterers. J. Quan. Spec. Rad. Transfer, 60, 309-324, https:// doi.org/10.1016/S0022-4073(98)00008-9.

Rose, C. R., and V. Chandrasekar, 2005: A system approach to GPM dual-frequency retrieval. IEEE Trans. Geosci. Remote Sens., 43, 1816-1826, https://doi.org/10.1109/TGRS.2005.851165.

Schwaller, M. R., and K. R. Morris, 2011: A ground validation network for the Global Precipitation Measurement mission. J. Atmos. Oceanic Technol., 28, 301-319, https://doi.org/10.1175/ 2010JTECHA1403.1.

Seto, S., and T. Iguchi, 2015: Intercomparison of attenuation correction methods for the GPM dual-frequency precipitation radar.J. Atmos. Oceanic Technol., 32, 915-926, https://doi.org/ 10.1175/JTECH-D-14-00065.1.

- — - and T. Oki, 2013: The basic performance of a precipitation retrieval algorithm for the Global Precipitation Measurement mission's single/dual frequency radar measurements. 
IEEE Trans. Geosci. Remote Sens., 51, 5239-5251, https:// doi.org/10.1109/TGRS.2012.2231686.

, T. Shimozuma, T. Iguchi, and T. Kozu, 2016: Spatial and temporal variations of mass-weighted mean diameter estimated by GPM/DPR. 2016 IEEE International Geoscience and Remote Sensing Symposium, Beijing, China, IEEE, 39383940, https://doi.org/10.1109/IGARSS.2016.7730023.

Skofronick-Jackson, G., and Coauthors, 2017: The Global Precipitation Measurement (GPM) mission for science and society. Bull. Amer. Meteor. Soc., 98, 1679-1695, https://doi.org/ 10.1175/BAMS-D-15-00306.1.

D. Kirschbaum, W. Petersen, G. Huffman, C. Kidd, E. Stocker, and R. Kakar, 2018: The Global Precipitation Measurement (GPM) mission's scientific achievements and societal contributions: Reviewing four years of advanced rain and snow observations. Quart. J. Roy. Meteor. Soc., 144, 27-48, https://doi.org/10.1002/qj.3313.

Testud, J., S. Oury, R. A. Black, P. Amayenc, and X. K. Dou, 2001: The concept of "normalized" distribution to describe raindrop spectra: A tool for cloud physics and cloud remote sensing.
J. Appl. Meteor., 40, 1118-1140, https://doi.org/10.1175/15200450(2001)040<1118:TCONDT $>2.0$.CO;2.

Thurai, M., G. J. Huang, V. N. Bringi, W. L. Randeu, and M. Schönhuber, 2007: Drop shapes, model comparisons, and calculations of polarimetric radar parameters in rain. J. Atmos. Oceanic Technol., 24, 1019-1032, https:/doi.org/10.1175/ JTECH2051.1.

Ulbrich, C. W., 1983: Natural variations in the analytical form of the raindrop size distribution. J. Appl. Meteor. Climatol., 22, 1764-1775, https://doi.org/10.1175/1520-0450(1983)022<1764: NVITAF $>2.0 . \mathrm{CO} ; 2$.

Williams, C. R., and Coauthors, 2014: Describing the shape of raindrop size distributions using uncorrelated raindrop mass spectrum parameters. J. Appl. Meteor. Climatol., 53, 12821296, https://doi.org/10.1175/JAMC-D-13-076.1.

Wolff, D. B., D. A. Marks, E. Amitai, D. S. Silberstein, B. L. Fisher, A. Tokay, J. Wang, and J. L. Pippitt, 2005: Ground validation for the Tropical Rainfall Measuring Mission (TRMM). J. Atmos. Oceanic Technol., 22, 365-380, https://doi.org/ 10.1175/JTECH1700.1. 